\title{
Minimum time optimal synthesis for two level quantum systems
}

Cite as: J. Math. Phys. 56, 012106 (2015); https://doi.org/10.1063/1.4906137

Submitted: 08 October 2014 . Accepted: 07 January 2015 . Published Online: 23 January 2015

Francesca Albertini, and Domenico D'Alessandro
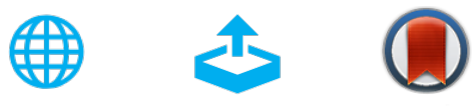

View Online

Export Citation

CrossMark

\section{ARTICLES YOU MAY BE INTERESTED IN}

Time minimal trajectories for a spin $1 / 2$ particle in a magnetic field

Journal of Mathematical Physics 47, 062101 (2006); https://

doi.org/10.1063/1.2203236

Optimal control in laser-induced population transfer for two- and three-level quantum systems

Journal of Mathematical Physics 43, 2107 (2002); https://doi.org/10.1063/1.1465516

A quantum engineer's guide to superconducting qubits

Applied Physics Reviews 6, 021318 (2019); https://doi.org/10.1063/1.5089550

Journal of

SIGN UP TODAY

Mathematical Physics

Receive the latest research updates 


\title{
Minimum time optimal synthesis for two level quantum systems
}

\author{
Francesca Albertini ${ }^{1, a)}$ and Domenico D'Alessandro $\left.{ }^{2, b}\right)$ \\ ${ }^{1}$ Dipartimento di Matematica, Università di Padova, Padova, Italy \\ ${ }^{2}$ Department of Mathematics, Iowa State University, Ames, Iowa 50011, USA
}

(Received 8 October 2014; accepted 7 January 2015; published online 23 January 2015)

\begin{abstract}
For the time optimal problem of an invariant system on $S U(2)$, with two independent controls and a bound on the norm of the control, the extremals of the Pontryagin maximum principle are explicit functions of time. We use this fact here to perform the optimal synthesis for these systems, i.e., to find all time optimal trajectories. Although the Lie group $S U(2)$ is three dimensional, time optimal trajectories can be described in the unit disk of the complex plane. We find that a circular trajectory separates optimal trajectories that reach the boundary of the unit disk from the others. Inside this separatrix circle, another trajectory (the critical trajectory) plays an important role in that all optimal trajectories end at an intersection with this curve. The results allow us to find the minimum time needed to achieve a given evolution of a two level quantum system. (C) 2015 AIP Publishing LLC. [http://dx.doi.org/10.1063/1.4906137]
\end{abstract}

\section{INTRODUCTION}

The control of quantum mechanical systems has offered further motivation to the study of control systems on Lie groups, and, in particular, on $S U(n)$ and its Lie subgroups, as the evolution of a closed quantum system can be often modeled as a right invariant system varying on one of such Lie groups (see, e.g., Refs. 1 and 7, and references therein). Among these models, systems on $S U(2)$ arguably represent the simplest non-trivial case, still a very rich one from a mathematical point of view. These two-level quantum systems are of fundamental interest in quantum physics and in quantum information, since they are the basic building block in the circuit based implementation of quantum information processing. ${ }^{21} \mathrm{~A}$ natural requirement in these implementations is to perform quantum operations (evolutions) in minimum time, both to shorten the overall time of computation and to avoid the detrimental effects of the interaction with the environment (de-coherence). For these reasons, these systems have been studied in many aspects and their (time) optimal control has been the subject of many papers (see, e.g., Refs. 4, 5, and 13-15, and references therein, see also 9, 10, 17, and 23 for the general optimal control problem for quantum mechanical systems and systems on Lie groups). Here, we add to this literature providing an explicit description of all time optimal trajectories for a system with two orthogonal controls $u_{x}$ and $u_{y}$ (cf. model (1) below) which are bounded as $u_{x}^{2}+u_{y}^{2} \leq \gamma^{2}$ at every time, with $|\gamma| \leq 1$. In particular, the model we consider is given by

$$
\dot{X}=\tilde{\sigma}_{z} X+u_{x} \tilde{\sigma}_{x} X+u_{y} \tilde{\sigma}_{y} X, \quad X(0)=\mathbf{1},
$$

where $X \in S U(2)$ and $\tilde{\sigma}_{x, y, z}$ are proportional to the Pauli matrices, $\sigma_{x, y, z}$, and form a basis of the Lie algebra $s u(2)$. They are defined as

$$
\tilde{\sigma}_{x}:=\frac{i}{2} \sigma_{x}=\frac{1}{2}\left(\begin{array}{cc}
0 & i \\
i & 0
\end{array}\right), \quad \sigma_{y}:=\frac{-i}{2} \sigma_{y}=\frac{1}{2}\left(\begin{array}{cc}
0 & -1 \\
1 & 0
\end{array}\right), \quad \sigma_{z}:=\frac{i}{2} \sigma_{z}=\frac{1}{2}\left(\begin{array}{cc}
i & 0 \\
0 & -i
\end{array}\right) .
$$

\footnotetext{
a) albertin@math.unipd.it

b)e-mail: daless@iastate.edu
} 
The Lie algebra $s u(2)$ is equipped with an inner product between matrices, $\langle\cdot, \cdot\rangle$, defined as $\langle A, B\rangle:=$ $\operatorname{Tr}\left(A B^{\dagger}\right)$, so that the associated norm is $\|A\|:=\sqrt{\langle A, A\rangle}$. With these definitions, the norm of each of the matrices $\tilde{\sigma}_{x, y, z}$ is $\frac{1}{\sqrt{2}}$.

We want to find, for every final condition $X_{f} \in S U(2)$, the controls $u_{x}, u_{y}$, that steer the state of system (1) from the identity, $\mathbf{1}$, to $X_{f}$ in minimum time, with the requirement that $u_{x}^{2}+u_{y}^{2} \leq \gamma^{2}$, $\gamma^{2} \leq 1$.

From a geometric point of view, model (1) represents a system on $S U(2)$ where one vector field is constant and an orthogonal vector field is allowed to vary with time but has a bounded norm. This model is of fundamental interest in quantum physics as it represents the control of a two level quantum systems, such as in $\mathrm{NMR}^{11,19}$ or quantum dots applications. ${ }^{8}$ In this respect, we remark that since quantum mechanical states are defined up to a phase factor driving to $+X_{f}$ and driving to $-X_{f}$ is equivalent from a physical point of view. However, since we will describe a method to obtain the minimum time control for any final condition, for a given desired transformation $\pm X_{f}$, we can compare the minimum time for $X_{f}$ and the minimum time for $-X_{f}$ and choose the smallest one of the two and the corresponding optimal control. The minimum time above described is also of interest in the general theory of Lie groups given the role of $s u(2)$ as the basic building block in Lie algebra theory. For example, one could devise suboptimal strategies for $S U(n)$ after a Cartan decomposition ${ }^{16}$ of the target element in factors which are in $S U(2)$.

Remark 1.1. The more general time optimal control problem for the system

$$
\dot{U}= \pm \omega_{0} \tilde{\sigma}_{z} U+v_{x} \tilde{\sigma}_{x} U+v_{y} \tilde{\sigma}_{y} U, \quad U(0)=\mathbf{1},
$$

with $\omega_{0}>0$, and $v_{x}^{2}+v_{y}^{2} \leq \omega_{0}^{2} \gamma^{2}$ can be reduced to the problem for system (1). Define $X(\tau):=$ $U\left(\frac{\tau}{\omega_{0}}\right)$, and new controls $u_{x, y}(\tau):=\frac{1}{\omega_{0}} v_{x, y}\left(\frac{\tau}{\omega_{0}}\right)$, then $X(\tau)$ solves

$$
\dot{X}= \pm \tilde{\sigma}_{z} X+u_{x} \tilde{\sigma}_{x} X+u_{y} \tilde{\sigma}_{y} X, \quad U(0)=\mathbf{1} .
$$

Thus, once the minimum time problem for (4) is solved with controls $u_{x}$ and $u_{y}$ and minimum time $T$, and $u_{x}^{2}+u_{y}^{2} \leq \gamma^{2}$, the original optimal control for (3) is solved with $v_{x, y}(\tau)=\omega_{0} u_{x, y}\left(\omega_{0} \tau\right)$, in time $\frac{T}{\omega_{0}}$ to drive to the same final condition. The optimal control problem for system (4) is the same as the one we have stated in the case + . In the case - it can be reduced to it. Assume we have solved the minimum time problem for system (1) for the final condition $X_{f}^{-1}$ and with controls $u_{x}$ and $u_{y}$ over an interval $[0, T]$. Then, it is easily verified that the control $-u_{x}(T-\tau),-u_{y}(T-\tau)$ over the same interval $[0, T]$ solves the problem of driving the state $X$ of system (4) with the - from the identity to $X_{f}$, in minimum time.

The paper is organized as follows. In Sec. II, we will describe methods to parametrize elements in $S U(2)$, and prove a simple property of the control system (1) which will allow us to consider only two parameters for the elements of $S U(2)$ rather than three when studying time optimal trajectories. In view of these facts, we will be able to perform the whole geometric analysis in the unit disk of the complex plane. In this section, we also recall how to apply the Pontryagin maximum principle of optimal control in this case and the form of the candidate time optimal controls and trajectories. In Sec. III, we solve the time optimal control problem for diagonal operators. As a limit of these trajectories, we identify a particular optimal trajectory which is a circle and plays a fundamental role for the whole analysis. All optimal trajectories leading to diagonal operators are outside this circle while all others are inside. Therefore, we call this curve the separatrix. ${ }^{24}$ For the special case $\gamma=1$, the separatrix curve coincides with the trajectory corresponding to the SWAP operator. The optimal trajectories for points inside the separatrix are described in Sec. IV.Here, we give the general picture as a conjecture which is supported by theoretical results and simulations. In order to complete the proof though, we use the additional assumption $\gamma \geq \frac{1}{\sqrt{3}}$, which allows us a better control of the geometry of the trajectories and to obtain useful bounds. In Sec. V, we provide a discussion of the results and show how these lead to a simple method to find the optimal control once the final condition is chosen. In particular, we discuss the application to the optimal control of two level quantum systems. In this section, we also compare our results with other work on the control of systems on $S U(2)$ and two level quantum systems and in particular the recent papers (Refs. 13 and 14). 


\section{PARAMETRIZATION OF SU(2) AND GENERAL PROPERTIES OF THE MODEL}

\section{A. Parametrization of the final conditions in the optimal control problem}

It is well known that the Lie group $S U(2)$ is diffeomorphic to the sphere $S^{3} \subseteq \mathrm{R}^{4}$ and it is Liehomeomorphic to the Lie group of unit quaternions, $S H, x+y \vec{i}+c \vec{j}+d \vec{k}$, with $x^{2}+y^{2}+c^{2}+d^{2}=1$, the homeomorphism $\cong$ being given by

$$
[x+y \vec{i}+c \vec{j}+d \vec{k} \in S H] \cong\left[\left(\begin{array}{cc}
x+y i & -(c+i d) \\
(c-i d) & x-i y
\end{array}\right) \in S U(2)\right] .
$$

By writing $-(c+i d)=e^{i \phi} M$ and $x+i y=e^{i \psi} \sqrt{1-M^{2}}$, with $0 \leq M \leq 1, \psi, \phi \in[0,2 \pi)$, we can write any matrix $X_{f} \in S U(2)$ using the three parameters $\psi, \phi$, and $M$, as

$$
X_{f}:=\left(\begin{array}{cc}
e^{i \psi} \sqrt{1-M^{2}} & e^{i \phi} M \\
-e^{-i \phi} M & e^{-i \psi} \sqrt{1-M^{2}}
\end{array}\right) .
$$

We shall sometimes normalize the parameter $\psi$ and use instead the parameter $x_{\psi}$, defined as $x_{\psi}$ $:=\frac{\psi-\pi}{\pi}$, with $x_{\psi} \in[-1,1)$. The parameter $\phi$ of the final condition $X_{f}$ in (6) does not affect the time optimal control problem, in the sense that matrices that differ only by the parameter $\phi$ can be reached in the same minimum time. This is a consequence of the following proposition. ${ }^{25}$

Proposition 2.1. The minimum time to reach $X_{f} \in S U(2)$ is the same as the minimum time to reach $e^{\tilde{\sigma}_{z} \alpha} X_{f} e^{-\tilde{\sigma}_{z} \alpha}$, for any $\alpha \in \mathrm{R}$.

Proof. Let $u_{x}$ and $u_{y}$ be optimal controls steering the state $X$ of (1) from the identity to $X_{f}$, in time $T_{o p t}$ and let $X_{o}:=X_{o}(\tau)$ be the corresponding trajectory. Define, for $j=x, y$, the constants $\beta_{j k}$ such that

$$
e^{\tilde{\sigma}_{z} \alpha} \sigma_{j} e^{-\tilde{\sigma}_{z} \alpha}=\sum_{k=x, y} \beta_{j, k} \tilde{\sigma}_{k}
$$

Define new controls $v_{x}, v_{y}$, for $k=x, y$, as $v_{k}:=\sum_{j=x, y} \beta_{j, k} u_{j}$. Moreover, notice that $v_{x}^{2}+v_{y}^{2}=u_{x}^{2}$ $+u_{y}^{2}$ so that, if $u_{x}, u_{y}$ is an admissible control so is $v_{x}, v_{y}$. With the control $v_{x}, v_{y}$, the trajectory solution of (1) is $U(\tau)=e^{\tilde{\sigma}_{z} \alpha} X_{o}(\tau) e^{-\tilde{\sigma}_{z} \alpha}$. In fact, differentiating $U(\tau)$ and using (1) for $X_{o}$ and (7), we obtain

$$
\begin{aligned}
& \dot{U}:=e^{\tilde{\sigma}_{z} \alpha} \dot{X} e^{-\tilde{\sigma}_{z} \alpha}=\tilde{\sigma}_{z} U+\left(\sum_{j=x, y} u_{j}\left(\sum_{k=x, y} \beta_{j, k} \tilde{\sigma}_{k}\right)\right) U= \\
& \tilde{\sigma}_{z} U+\left(\sum_{k=x, y}\left(\sum_{j=x, y} \beta_{j, k} u_{j}\right) \tilde{\sigma}_{k}\right) U=\tilde{\sigma}_{z} U+\left(\sum_{k=x, y} v_{k} \tilde{\sigma}_{k}\right) U .
\end{aligned}
$$

This shows that the optimal time to reach $e^{\tilde{\sigma}_{z} \alpha} X_{f} e^{-\tilde{\sigma}_{z} \alpha}$ is not greater than the one to reach $X_{f}$. By exchanging the roles of $X_{f}$ and $e^{\tilde{\sigma}_{z} \alpha} X_{f} e^{-\tilde{\sigma}_{z}^{\alpha}}$, the opposite is seen to be true. Therefore, the minimum time is the same in the two cases as stated.

Remark 2.2. The proof can be generalized with only formal modifications to more general systems on (Lie subgroups of) $S U(n)$ of the form $\dot{X}=A X+\sum_{j=1}^{m} u_{j} B_{j} X$. We can replace $e^{\tilde{\sigma} z^{\alpha}}$ with any matrix $K$ in (a Lie subgroup of) $S U(n)$, which commutes with $A$ and it is such that $\operatorname{span}\left\{K B_{1} K^{\dagger}, \ldots\right.$, $\left.K B_{m} K^{\dagger}\right\}=\operatorname{span}\left\{B_{1}, \ldots, B_{m}\right\}$.

In view of Proposition 2.1, the only element that is relevant to determine the minimum time to reach $X_{f}$ in (6) is the element $X_{1,1}$ in the matrix $X_{f}$. This will be parametrized by phase $\psi$ (or $x_{\psi}$ ) and magnitude $M$ or, more often, by its real and imaginary parts, i.e., as a point $x+i y$ in the unit disk of the complex plane. To every (optimal) trajectory in $S U(2)$, there corresponds a curve starting from $(1,0)$ in the unit disk. Points in the unit disk correspond to classes of matrices in $S U(2)$ which can be reached in the same minimum time, and differ only by the phase of the element $X_{1,2}$. 


\section{B. The Pontryagin maximum principle and the expression of optimal candidates}

Consider the problem of driving the state $X$ of (1) from the identity, $\mathbf{1}$, to a final condition $X_{f}$, with bound $u_{x}^{2}+u_{y}^{2} \leq \gamma^{2}$, in minimum time. The Pontryagin maximum principle states that, if $u_{x}, u_{y}$ is optimal, and $X_{o}$ is the optimal trajectory, then there exists a nonzero matrix $\tilde{M} \in \operatorname{su}(2)$, such that, for almost every time $\tau, u_{x}(\tau), u_{y}(\tau)$, are the values of $v_{x}$ and $v_{y}$, that maximize the Hamiltonian function

$$
H\left(\tilde{M}, X_{o}, v_{x}, v_{y}\right):=\left\langle\tilde{M}, X_{o}^{\dagger} \tilde{\sigma}_{z} X_{o}\right\rangle+v_{x}\left\langle\tilde{M}, X_{o}^{\dagger} \tilde{\sigma}_{x} X_{o}\right\rangle+v_{y}\left\langle\tilde{M}, X_{o}^{\dagger} \tilde{\sigma}_{y} X_{o}\right\rangle .
$$

Furthermore, $H\left(\tilde{M}, X_{o}(\tau), u_{x}(\tau), u_{y}(\tau)\right)$ is constant for almost every $\tau .^{26}$ Define, $b_{x, y, z}:=\langle\tilde{M}$, $\left.X_{o}^{\dagger} \tilde{\sigma}_{x, y, z} X_{o}\right\rangle$. The maximization condition implies that

$$
u_{x, y}=\gamma \frac{b_{x, y}}{\sqrt{b_{x}^{2}+b_{y}^{2}}}
$$

unless $b_{x}$ and $b_{y}$ are both zero, in which case the corresponding arc is called singular. Differentiating $b_{x, y, z}$ with respect to time, using (1), and the standard commutation relations for the matrices $\tilde{\sigma}_{x, y, z}$ in (2), ${ }^{27}$ we arrive at the following system of differential equations for $b_{x}, b_{y}$, and $b_{z}$ :

$$
\begin{gathered}
\dot{b}_{x}=b_{z} u_{y}-b_{y}, \\
\dot{b}_{y}=b_{x}-b_{z} u_{x}, \\
\dot{b}_{z}=b_{y} u_{x}-b_{x} u_{y} .
\end{gathered}
$$

On a nonsingular arc, given the expression of the controls $u_{x}$ and $u_{y}$ in (9), we have that $b_{z}$ is constant. This together with the fact that the Hamiltonian (8), which takes the form $H=b_{z}+\gamma \sqrt{b_{x}^{2}+b_{y}^{2}}$, is also constant, implies that the controls $u_{x}$ and $u_{y}$ (for nonsingular extremals) can be written as (cf., the solutions of (10) and (11))

$$
u_{x}=\gamma \sin (\omega \tau+\tilde{\phi}), \quad u_{y}=-\gamma \cos (\omega \tau+\tilde{\phi}),
$$

for some frequency $\omega \in \mathbf{R}$ and phase $\tilde{\phi} \in \mathbf{R} .^{28}$ For singular arcs where $b_{x} \equiv b_{y} \equiv 0$, from (12) $b_{z}$ $=$ const $\neq 0$ which $^{29}$ therefore gives from (10), (11), $u_{x} \equiv 0, u_{y} \equiv 0$. Therefore, singular arcs starting from a point $X_{1}$ have the form $e^{\tilde{\sigma}_{z} \tau} X_{1}$, for $\tau \in\left[0, \tau_{1}\right]$ for some $\tau_{1}>0$. In general, the optimal trajectory may be an alternation of singular and nonsingular arcs. We shall see, however, in Theorem 1 and its proof that singular arcs are never optimal. ${ }^{30}$

Focusing on nonsingular arcs, using the controls (13) in (1), the resulting differential equation can be explicitly integrated (see, e.g., Ref. 6, p. 446). Direct verification shows that the solution is given by

$$
X(\tau, \omega, \tilde{\phi}):=\left(\begin{array}{cc}
e^{i \omega t}\left(\cos (a t)+i \frac{b}{a} \sin (a t)\right) & e^{i(\omega t+\tilde{\phi})} \frac{\gamma}{a} \sin (a t) \\
-e^{-i(\omega t+\tilde{\phi})} \frac{\gamma}{a} \sin (a t) & e^{-i \omega t}\left(\cos (a t)-i \frac{b}{a} \sin (a t)\right)
\end{array}\right),
$$

for $t ;=\frac{\tau}{2}, b:=1-\omega, a:=\sqrt{\gamma^{2}+b^{2}}$. For given $\omega$ and $\tilde{\phi}$, the time $T$ is the minimum time to reach $X_{f}$ if $X_{f}:=X(T, \omega, \tilde{\phi})$ and there is no smaller $T_{1}$ and pair $\omega_{1}$ and $\tilde{\phi}_{1}$ such that $X_{f}:=X\left(T_{1}, \omega_{1}, \tilde{\phi}_{1}\right)$.

In the expression (14), the phase of the element $(1,2)$ does not affect the (minimum) time to reach a given target, in the sense that we can always "tune" $\tilde{\phi}$ to give an arbitrary phase to the $(1,2)$ element of the final condition. ${ }^{31}$

In the following, when we shall refer to "time" we shall refer to $t: \frac{\tau}{2}$. Curves that satisfy the Pontryagin maximum principle are called extremal curves.

\section{Properties of extremal curves}

Any optimal candidate is represented by a parametric curve in the complex plane, and in particular inside the unit disk, which starts from the point $(1,0)$ and represents the $X_{1,1}$ element of the trajectory of (1). If they do not contain singular arcs, these curves can be parametrized by the frequency $\omega$ of 
the optimal control candidates while the phase does not play any role, as it only affects the phase of the $X_{1,2}$ element and can be arbitrarily tuned. From (14), the nonsingular trajectories are explicitly given by

$$
\begin{aligned}
& x(t):=x_{\omega}(t)=\cos (\omega t) \cos (a t)-\frac{b}{a} \sin (\omega t) \sin (a t), \\
& y(t):=y_{\omega}(t)=\sin (\omega t) \cos (a t)+\frac{b}{a} \cos (\omega t) \sin (a t),
\end{aligned}
$$

with $b:=1-\omega, a=\sqrt{b^{2}+\gamma^{2}}$.

We also have (cf. (14)) for the distance, $r$, of the point from the origin,

$$
1-M^{2}(t):=r^{2}(t):=x_{\omega}^{2}(t)+y_{\omega}^{2}(t)=1-\frac{\gamma^{2}}{a^{2}} \sin ^{2}(a t) .
$$

The phase $\psi(t)$ is given (cf. (14)) for $0 \leq t<\frac{\pi}{2 a}$ by

$$
\psi(t)=\omega t+\arctan \left(\frac{b}{a} \tan (a t)\right)
$$

and for $\frac{\pi}{2 a}<t \leq \frac{\pi}{a}$,

$$
\psi(t)=\omega t+\pi+\arctan \left(\frac{b}{a} \tan (a t)\right) .
$$

There will be some values of the frequency $\omega$ which play an important role in the analysis that follows. We define them at the outset. In particular, we define $\omega^{*}:=\frac{1+\gamma^{2}}{2}, \omega_{c}:=2 \omega^{*}=1+\gamma^{2}$. Correspondingly, we define $b^{*}:=1-\omega^{*}, b_{c}:=1-\omega_{c}, a^{*}:=\sqrt{\gamma^{2}+\left(b^{*}\right)^{2}}=\frac{1+\gamma^{2}}{2}=\omega^{*}$, and $a_{c}:=\sqrt{\gamma^{2}+\left(b_{c}\right)^{2}}$ $=\gamma \sqrt{\gamma^{2}+1}$.

We record few properties of the extremal trajectories.

Fact 1: From Eq. (17), we have

$$
\frac{d r^{2}}{d t}=\frac{-2 \gamma^{2} \sin (2 a t)}{a}
$$

which implies that $r(t)$ is decreasing for $t \in\left(0, \frac{\pi}{2 a}\right)$, and it is increasing for $t \in\left(\frac{\pi}{2 a}, \frac{\pi}{a}\right)$. At the time $t=\frac{\pi}{a}$, the trajectory reaches the boundary of the unit disk. Moreover, since $\left.\frac{d\left(r^{2}\right)}{d t}\right|_{t=0}=0,\left.\frac{d^{2}\left(r^{2}\right)}{d t^{2}}\right|_{t=0}=$ $-2 \gamma^{2},\left.\frac{d^{3}\left(r^{2}\right)}{d t^{3}}\right|_{t=0}=0$, and $\left.\frac{d^{4}\left(r^{2}\right)}{d t^{4}}\right|_{t=0}=8 a^{2} \gamma^{2}$, we have that given $\omega_{1}$ and $\omega_{2}$ and letting $a_{1,2}$ and $r_{1,2}(t)$ the corresponding value for the constant $a$ and $r(t)$, if $a_{1}>a_{2}$, for $t$ in a neighborhood of 0 , we have $r_{1}(t)>r_{2}(t)$.

Fact 2: Calculating $\frac{d \psi}{d t}$ from (18), (19), after some algebra, we obtain

$$
\frac{d \psi}{d t}=\frac{-\gamma^{2} \sin ^{2}(a t) \omega+a^{2}}{a^{2} \cos ^{2}(a t)+b^{2} \sin ^{2}(a t)} .
$$

Equation (20) implies that for $\omega \leq 1$ the phase is always increasing. Moreover, when $\omega>0$, we have

$$
-\gamma^{2} \sin ^{2}(a t) \omega+a^{2}=\omega^{2}-\left(2+\gamma^{2} \sin ^{2}(a t)\right) \omega+\left(\gamma^{2}+1\right) \geq \omega^{2}-\left(2-\gamma^{2}\right) \omega+\left(\gamma^{2}+1\right) .
$$

Since the last polynomial is positive when $\omega>\omega_{c}:=1+\gamma^{2}$, we derive that the phase is always increasing for $\omega \leq 1$ and for $\omega \geq \omega_{c}$.

Fact 3: The fully singular curve corresponds to the boundary of the unit disk. Therefore, every point in the interior of the unit disk must be reached by an optimal trajectory which contains a nonsingular arc. We shall in fact see in Theorem 1 that even for the points on the boundary the optimal trajectories are nonsingular, and this implies that all the optimal trajectories do not contain singular arcs.

Fact 4: (Principle of optimality) If a trajectory reaching a point $P$ is optimal, then that trajectory is optimal for every point on that curve before $P$.

Fact 5: When two nonsingular trajectories intersect at a point $P$, they cannot be both optimal after the point $P$. In fact, if they reach $P$ at different times, then, obviously, the one that reaches $P$ at 
a greater time is not optimal. If they reach $P$ at the same time, then we could possibly switch from one value of $\omega$ to the other in the control and still have an optimal trajectory. This contradicts the fact that all the nonsingular extremals have the form (13). If a curve is optimal for every point before a point $P$ and not optimal after $P$ we say that a curve looses optimality at $P$.

Fact 6: Because of the existence of the optimal control under our assumptions (see, e.g., Ref. 12), and the fact we will see in Theorem 1 that only fully nonsingular extremal is optimal, every point in the unit disk is reached by at least one fully nonsingular curve and among those that reach the point at least one is optimal.

\section{OPTIMAL CONTROL PROBLEM FOR DIAGONAL FINAL CONDITIONS AND THE SEPARATRIX CURVE}

\section{A. Diagonal operators}

Assume the final condition $X_{f}:=\left(\begin{array}{cc}e^{i \psi_{f}} & 0 \\ 0 & e^{-i \psi_{f}}\end{array}\right)$, that is, we want to drive in minimum time to a point on the boundary of the unit disk. According to formula (17), extremal nonsingular trajectories reach the boundary of the unit disk at times $T=\frac{k \pi}{a}$. If $T$ is the final time in (14), we have the two equations

$$
\begin{aligned}
T & =\frac{k \pi}{a}, \quad k \geq 0, \\
\omega T+a T & =\psi_{f}+2 m \pi, \quad m \in \mathbb{Z},
\end{aligned}
$$

which give the condition on the norm of the off diagonal term and on the phase of the diagonal term, respectively. ${ }^{32}$ Plugging (21) into (22), we have

$$
k \pi\left(1+\frac{\omega}{a}\right)=\psi_{f}+2 m \pi .
$$

A study of the function $f(\omega):=\frac{\omega}{a}$ for $\omega \in(-\infty, \infty)$ reveals that this function is bounded below by -1 , so that, when $\psi_{f} \in(0,2 \pi)$, (23) can only be verified for $m \geq 0$. In general, we say that a pair $k, m$ is feasible if there exists a $T \geq 0$, such that (21) and (23) are verified. We denote this (necessarily unique) $T$ by $T_{k, m}$. Notice that not all pairs $k>0, m \geq 0$ are feasible (the function $\frac{\omega}{a}$ is bounded). We shall show that no matter what $\psi_{f} \in(0,2 \pi)$ is, the minimum of these times is $T_{1,0}$ which is feasible. ${ }^{33}$ The proof can be achieved in two steps given by the following two lemmas. The result for the diagonal case is summarized in Theorem 1. Proofs of Lemmas 3.1 and 3.2 are given in Appendix A.

Lemma 3.1. For every $k>0$ and $m>0$,

$$
T_{k, m} \geq T_{k, 0}
$$

Lemma 3.2. For every $k>0$,

$$
T_{k, 0} \geq T_{1,0}
$$

Theorem 1. Assume $\gamma \leq 1$. Then the minimum time to reach a diagonal operator $X_{f}:=$ $\left(\begin{array}{cc}e^{i \psi_{f}} & 0 \\ 0 & e^{-i \psi_{f}}\end{array}\right), \psi_{f} \in(0,2 \pi)$, is

$$
T_{\min }=T_{1,0}\left(\psi_{f}\right):=\frac{\psi_{f}\left(2 \pi-\psi_{f}\right)}{\pi-\psi_{f}+\sqrt{\pi^{2}+\gamma^{2} \psi_{f}\left(2 \pi-\psi_{f}\right)}},
$$

which is obtained with the controls (13), with $\tilde{\phi}$ arbitrary and $\omega$ given by ${ }^{34}$

$$
\omega=\frac{x_{\psi_{f}}}{1-x_{\psi_{f}}^{2}}\left(-x_{\psi_{f}}+\sqrt{1+\gamma^{2}\left(1-x_{\psi_{f}}^{2}\right)}\right) .
$$


Proof. The theorem summarizes the previous two Lemmas. The expression of the optimal frequency $\omega$ is obtained from (23), (A8), with $k=1$ and $m=0$.

To make sure that this time is optimal, we need to compare it with the one obtained with the singular trajectory which is $T_{\text {sing }}\left(\psi_{f}\right)=\psi_{f}$. In fact, we have $T_{\min }<T_{\text {sing }}$. This follows from

$$
\frac{T_{\min }}{\psi_{f}}=\frac{2 \pi-\psi_{f}}{\pi-\psi_{f}+\sqrt{\pi^{2}+\gamma^{2} \psi_{f}\left(2 \pi-\psi_{f}\right)}}<1=\frac{T_{\text {sing }}}{\psi_{f}} .
$$

A consequence of this theorem, which we already mentioned before, is that no optimal trajectory may contain a singular arc. The same state transfer corresponding to the singular arc can be obtained in smaller time.

\section{B. The separatrix curve}

Reconsider formula (27). There is a one to one correspondence between values of $x_{\psi_{f}} \in(-1,1)$ (alternatively values of $\left.\psi_{f} \in(0,2 \pi)\right)$ and values of $\omega \in\left(-\infty, \omega^{*}\right)$. In fact, the right hand side of (27) is a strictly increasing function of $x_{\psi_{f}}$ with

$$
\lim _{x_{\psi_{f}} \rightarrow-1} \omega=\lim _{\psi_{f} \rightarrow 0} \omega=-\infty
$$

and

$$
\lim _{x_{\psi_{f} \rightarrow 1}} \omega=\lim _{\psi_{f} \rightarrow 2 \pi} \omega=\frac{1+\gamma^{2}}{2}=\omega^{*} .
$$

Consider now the trajectory corresponding exactly to $\omega=\omega^{*}=\frac{1+\gamma^{2}}{2}$. In this case, since $\omega^{*}=a^{*}$, the parametric equations (15) and (16) become

$$
\begin{aligned}
& x_{\omega}(t)=\frac{2}{1+\gamma^{2}} \cos \left(\omega^{*} t\right)-\frac{1-\gamma^{2}}{1+\gamma^{2}}, \\
& y_{\omega}(t)=\frac{2}{1+\gamma^{2}} \cos \left(\omega^{*} t\right) \sin \left(\omega^{*} t\right) .
\end{aligned}
$$

These equations represent a circle with center in

$$
P=\left(\frac{\gamma^{2}}{1+\gamma^{2}}, 0\right)
$$

and radius $\frac{1}{1+\gamma^{2}}$. We shall call this circle the "separatrix." The following lemma justifies this name.

Lemma 3.3. All the optimal trajectories corresponding to diagonal operators (described in Subsection III A) for $t \leq \frac{\pi}{a}$, intersect the separatrix curve only in the point $(1,0)$.

The proof is in Appendix A.

Figures 1 and 2 give some plots of the trajectories outside the separatrix, leading to diagonal operators for the cases $\gamma=\frac{1}{2}$ and $\gamma=1$, respectively. The separatrix is the red circle in both cases. The cases $\omega=-3, \omega=0$, and $\omega=\frac{1}{2}$ are displayed explicitly for $\gamma=\frac{1}{2}$ and the same values of $\omega$ 's and $\omega=\frac{8}{9}$ are displayed for $\gamma=1$. As $\omega \rightarrow \omega^{*}$, the trajectories tend to the separatrix.

The following proposition states two important properties of the optimal trajectories outside the separatrix.

\section{Proposition 3.4.}

1. The trajectories corresponding to $\omega \in\left(-\infty, \omega^{*}\right)$ loose optimality after reaching the boundary of the unit disk. 


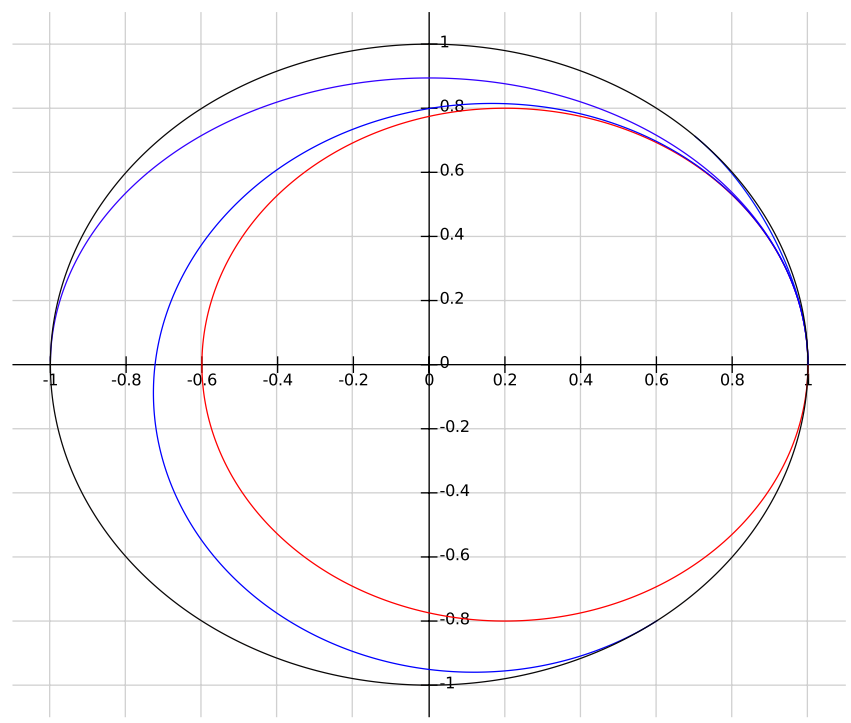

FIG. 1. Optimal trajectories (in blue) to reach the boundary of the unit disk for various values of $\omega$ and $\gamma=\frac{1}{2}$. The outermost trajectory is the one corresponding to $\omega=-3$, the next one (reaching the point $(-1,0)$ ) corresponds to $\omega=0$. The innermost trajectory is the one corresponding to $\omega=\frac{1}{2}$. The separatrix is the red circle centered at the point $\left(\frac{1}{5}, 0\right)$

2. Every point outside the separatrix is reached by an optimal trajectory (before reaching the boundary) corresponding to a single value of $\omega$, with $\omega \in\left(-\infty, \omega^{*}\right)$.

Proof. To prove 1., recall from Fact 2 of Subsection II C that the phase $\psi$ is always increasing, since $\omega^{*} \leq 1$. This means that any of the trajectories corresponding to $\omega \in\left(-\infty, \omega^{*}\right)$ after hitting the boundary will necessarily intersect another trajectory corresponding to a larger value of (final) $\psi_{f}$ which is optimal. Therefore, such a trajectory looses optimality at the boundary.

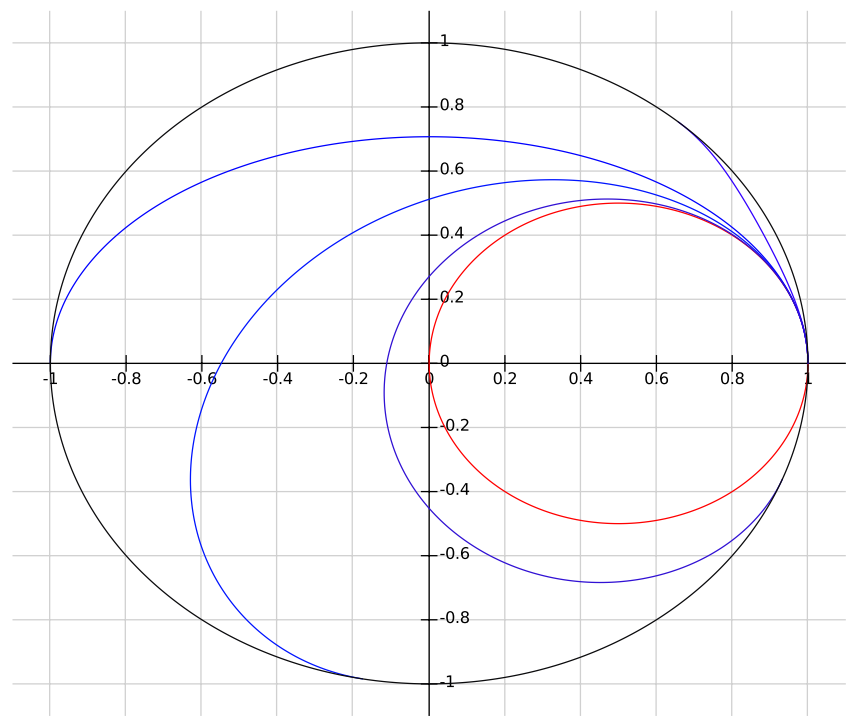

FIG. 2. Optimal trajectories (in blue) to reach the boundary of the unit disk for various values of $\omega$ and $\gamma=1$. The outermost trajectory is the one corresponding to $\omega=-3$, the next one (reaching the point $(-1,0)$ ) corresponds to $\omega=0$. The next on is the one corresponding to $\omega=\frac{1}{2}$. The innermost trajectory is the one corresponding to $\omega=\frac{8}{9}$. The separatrix is the red circle centered at the point $\left(\frac{1}{2}, 0\right)$. 
To prove 2., consider a point $P$ outside the separatrix and assume by contradiction that none of the curves reaching the boundary and corresponding to $\omega \in\left(-\infty, \omega^{*}\right)$ contains such a point. In particular, denote by $C_{\psi_{f}}$ any such curve corresponding to the phase $\psi_{f} \in(0,2 \pi)$. By the existence of the optimal control for $P$, there must exist an optimal trajectory ending in $P$, which we denote by $C_{P}$, defined in $\left[0, t_{P}\right]$, with $t_{P}<\frac{\pi}{a}$ (cf. (17)). Any of the trajectories $C_{\psi_{f}}$ and $C_{P}$ never intersect (except for the point $(1,0)$ ). Express the trajectory $C_{\psi_{f}}$ and $C_{P}$ as polar equations $r=r(\psi)$ with $\psi$ the (variable) phase. In particular, we write $r=r_{f}(\psi)$ for $C_{\psi_{f}}$ and $r=r_{P}(\psi)$ for $C_{P}$. With this notation, we say that $C_{\psi_{f}}$ is above $C_{P}$ if $r_{f}(\psi)$ is greater than $r_{P}(\psi)$ for one (and therefore all since they cannot intersect) $\psi \neq 0$ which are in the common domain of the function $r_{f}$ and $r_{P}$. Analogously, we say that $C_{\psi_{f}}$ is below $C_{P}$ if $r_{f}(\psi)$ is smaller than $r_{P}(\psi)$. Consider the set $A_{P}\left(B_{P}\right)$ of all $\psi_{f} \in(0,2 \pi)$ which are such that $C_{\psi_{f}}$ is above (below) $C_{P}$. It is important to notice that both $A_{P}$ and $B_{P}$ are not empty. $A_{P}$ is not empty because it definitely contains all $\psi_{f}$ 's smaller than the phase of $P$ since the phase is always increasing from formula (20). $B_{P}$ is not empty because it is enough to take a curve $C_{\psi_{f}}$ sufficiently close to the separatrix to leave $P$ on the right. Moreover, $A_{P} \cup B_{P}=(0,2 \pi)$. By continuity (again using the fact that $C_{\psi_{f}}$ and $C_{P}$ never intersect), $A_{P}$ and $B_{P}$ are both open set. Since they are not empty, this contradicts the connectedness of $A_{P} \cup B_{P}=(0,2 \pi)$.

\section{The SWAP operator}

The SWAP operator, is the operator that in quantum information theory, corresponds to a logic operation NOT. It inverts the state of a two level quantum system. It is given in the computational basis by

$$
X_{S W A P}:=\left(\begin{array}{cc}
0 & 1 \\
-1 & 0
\end{array}\right),
$$

which corresponds to the origin of the unit disk. In formula (14), we need $a=\gamma, b=0$, and $\omega=1$ (resonance condition ${ }^{3}$ ) and, minimum time $T_{\min }\left(X_{S W A P}\right)=\frac{\pi}{2 \gamma}$. The optimal trajectory is

$$
X(t)=\left(\begin{array}{cc}
e^{i t} \cos (\gamma t) & e^{i(t+\tilde{\phi})} \sin (\gamma t) \\
-e^{-i(t+\tilde{\phi})} \sin (\gamma t) & e^{-i t} \cos (\gamma t)
\end{array}\right) .
$$

We have that if $\gamma<1$, then $\omega^{*}=\frac{1+\gamma^{2}}{2}<1$, and the optimal trajectory corresponding to the SWAP operator is inside the separatrix. It turns out (see Theorem 2 ) that this trajectory is going to be optimal until it reaches a critical curve corresponding to $\omega=\omega_{c}=2 \omega^{*}$ (this curve will be precisely defined in Sec. IV). When $\gamma=1, \omega^{*}=1$, the optimal trajectory corresponding to the SWAP operator coincides with the separatrix.

\section{THE OPTIMAL CONTROL PROBLEM INSIDE THE SEPARATRIX}

From now on we denote by $\mathcal{S}$ the closed region inside the separatrix. For points in $\mathcal{S}$, the frequency $\omega$ of the optimal control must be greater than or equal to $\omega^{*}=\frac{1+\gamma^{2}}{2}$. In fact, as we have seen in Sec. III, the trajectories corresponding to $\omega<\omega^{*}$ do not intersect the separatrix before touching the boundary of the unit disk (Lemma 3.3) and, after touching the boundary of the unit disk, they are not optimal anymore (Proposition 3.4). Therefore, for all points in $\mathcal{S}$, the optimal trajectories are with $\omega \geq \omega^{*}$.

A particularly important role is played by the curve corresponding to $\omega_{c}:=2 \omega^{*}:=\gamma^{2}+1$. This curve presents a cuspid point, i.e., a point where both $\dot{x}$ and $\dot{y}$ are zero. In particular, recalling the definition of $a_{c}$ as the value of $a$ corresponding to $\omega_{c}$, i.e., $a_{c}:=\gamma \sqrt{1+\gamma^{2}}$, from (15) and (16), we obtain

$$
\begin{gathered}
\dot{x}_{\omega_{c}}(t)=-\sin \left(\omega_{c} t\right) \cos \left(a_{c} t\right), \\
\dot{y}_{\omega_{c}}(t)=\cos \left(\omega_{c} t\right) \cos \left(a_{c} t\right),
\end{gathered}
$$


and both derivatives are zero when $t=\frac{\pi}{2 a_{c}}$. We shall call the trajectory corresponding to $\omega=\omega_{c}$ until the point corresponding to $t=\frac{\pi}{2 a_{c}}$, the critical trajectory. Its final point is

$$
x_{\omega_{c}}\left(\frac{\pi}{2 a_{c}}\right)=\frac{\gamma}{\sqrt{1+\gamma^{2}}} \sin \left(\pi \frac{\sqrt{1+\gamma^{2}}}{2 \gamma}\right), \quad y_{\omega_{c}}\left(\frac{\pi}{2 a_{c}}\right)=-\frac{\gamma}{\sqrt{1+\gamma^{2}}} \cos \left(\pi \frac{\sqrt{1+\gamma^{2}}}{2 \gamma}\right) .
$$

It is in particular a point on the circle centered at the origin with radius $\frac{\gamma}{\sqrt{1+\gamma^{2}}}$. Such a circle will play a role in the analysis that follows. We call it the critical circle.

The general picture of the optimal synthesis for points in $\mathcal{S}$ is presented in Theorem 2. We believe this theorem holds for general values of $\gamma \leq 1$ but we were able to completely prove it only for $\gamma \in\left[\frac{1}{\sqrt{3}}, 1\right]$.

Theorem 2. Assume $\gamma \in\left[\frac{1}{\sqrt{3}}, 1\right]$. The only optimal trajectories for points in $\mathcal{S}$ correspond to $\omega \in\left[\omega^{*}, \omega_{c}\right]$.

1. The trajectory corresponding to $\omega_{c}$ until the point (36) is optimal for the points on the critical trajectory.

2. The trajectory corresponding to $\omega^{*}$ is optimal for the points of the separatrix.

3. For any other point in $\mathcal{S}$, there exists a unique value of $\omega \in\left(\omega^{*}, \omega_{c}\right)$ and an optimal trajectory corresponding to $\omega$ leading to that point.

From the theorem, it follows that both the separatrix and the critical trajectory are optimal. The trajectories corresponding to values of $\omega \in\left(\omega^{*}, \omega_{c}\right)$ are optimal until they intersect the critical trajectory. The situation is described in Figure 3 for the case $\gamma=1$ and Figure 4 for the case $\gamma=\frac{1}{2}$, respectively. In both figures, the red circle is the separatrix and the black trajectory inside the separatrix is the critical trajectory. Optimal trajectories depicted in blue start from the point $(1,0)$ and end, loosing optimality, on the critical trajectory.

Before giving the proof of Theorem 2, we need to establish some preliminary results. Some proofs are presented in Appendix B. Here, we give the main ideas highlighting where the assumption $\gamma \geq \frac{1}{\sqrt{3}}$ is used. The following property makes the geometry of the problem easier to visualize.

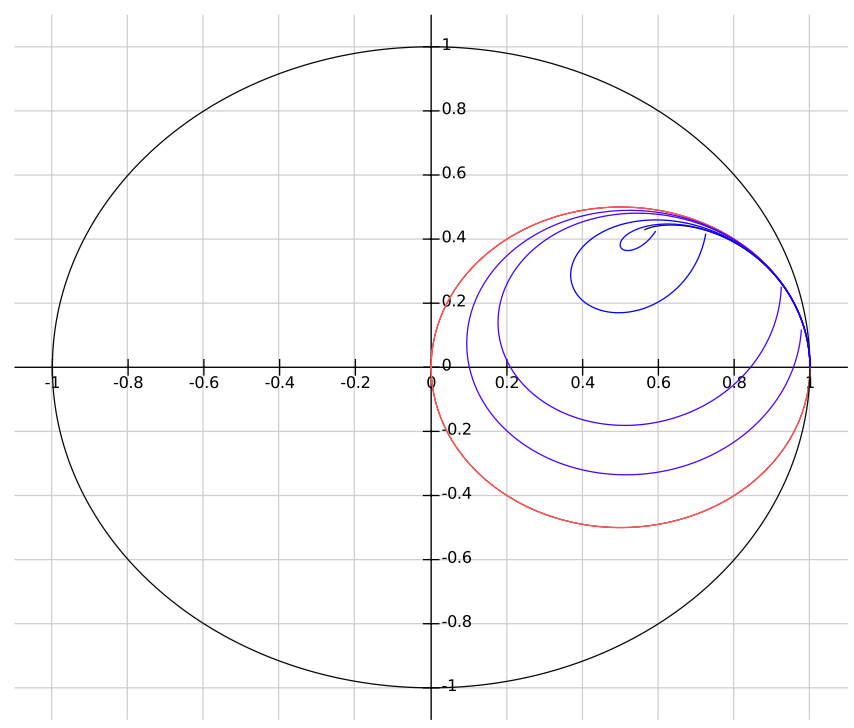

FIG. 3. Optimal trajectories inside the separatrix (in red) for $\gamma=1$. The critical trajectory is in black, while the trajectories for $\omega=1.1 \omega^{*}, \omega=1.2 \omega^{*}, \omega=1.5 \omega^{*}, \omega=1.8 \omega^{*}$, are in blue (starting closer to the separatrix when $\omega \rightarrow \omega^{*}=1$ and starting closer to the critical trajectory when $\omega \rightarrow \omega_{c}=2$ ). 


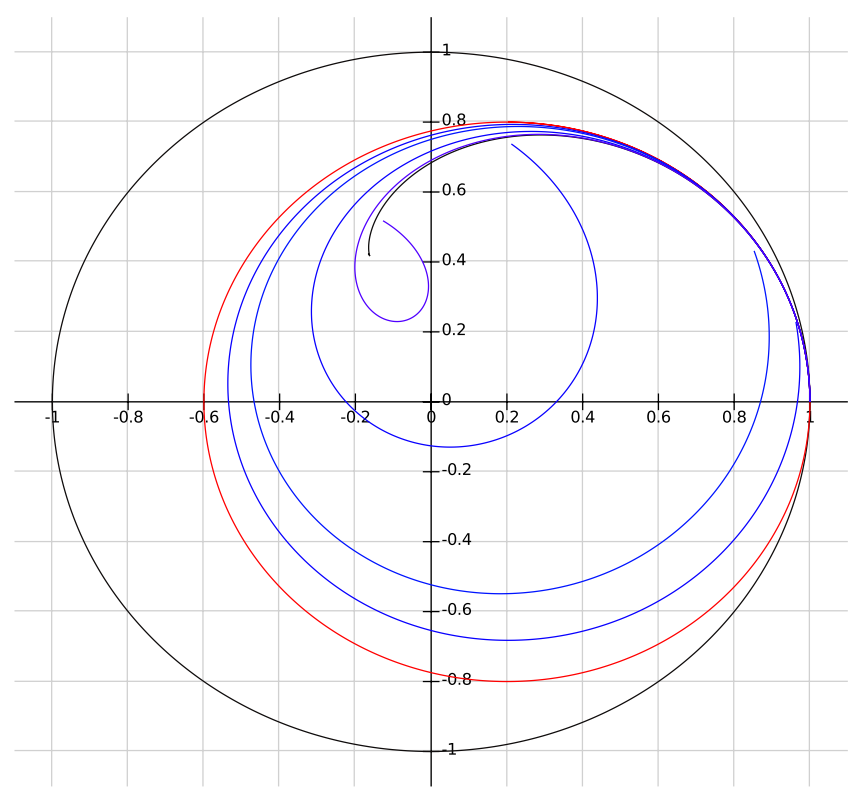

FIG. 4. Optimal trajectories inside the separatrix (in red) for $\gamma=\frac{1}{2}$. The critical trajectory is in black, while the trajectories for $\omega=1.1 \omega^{*}, \omega=1.2 \omega^{*}, \omega=1.5 \omega^{*}, \omega=1.8 \omega^{*}$, are in blue (starting closer to the separatrix when $\omega \rightarrow \omega^{*}=\frac{1+\gamma^{2}}{2}=\frac{1+\frac{1}{4}}{2}$ and starting closer to the critical trajectory when $\omega \rightarrow 2 \omega^{*}=1+\gamma^{2}=\frac{5}{4}$ ).

Proposition 4.1. Assume $\gamma \geq \frac{1}{\sqrt{3}}$. The whole critical trajectory is in the interior of the first quadrant, except (possibly) at the endpoints.

Proof. Studying the velocity (34), (35), we check that, for the given parameters, and under the assumption $\gamma \geq \frac{1}{\sqrt{3}}$ the derivative of $x_{\omega_{c}}$ is always negative until $\frac{\pi}{2 a_{c}}$, so the minimum is obtained at the point (36), which is such that the $x$ coordinate is non-negative. Since $y_{\omega_{c}}$ is always positive for $t \neq 0, t \leq \frac{\pi}{2 a_{c}}$ the claim follows.

When $\gamma<\frac{1}{\sqrt{3}}$ this is not verified, for example, when $\gamma=\frac{1}{2}$ we see in Figure 4 that the critical curve has a portion in the second quadrant. In general, the critical trajectory looks like a spiral winding around the origin more and more times as $\gamma \rightarrow 0$.

Consider the critical trajectory. Starting from the point $(1,0)$, the distance from the origin decreases monotonically according to formula (17) and the last point is on the critical circle. We introduce a parameter $\lambda$ to parametrize the critical trajectory. For any $t \in\left[0, \frac{\pi}{2 a_{c}}\right]$, let

$$
\lambda=\sin \left(a_{c} t\right) .
$$

Then, $\lambda \in[0,1]$. Because of (17), $\lambda$ indicates the distance of the point on the critical trajectory from the origin, which goes from 1 to $\sqrt{1-\frac{\gamma^{2}}{a_{c}^{2}}}=\frac{\gamma}{\sqrt{1+\gamma^{2}}}$ (cf. (36)). For a given value of $\lambda$, i.e., for points on the same circle centered at the origin, we compare the phase of any trajectory (corresponding to a given value of $\omega$ and $a$ ) with the phase for the critical trajectory. In doing so, we assume $0 \leq t<\frac{\pi}{2 a}$ and we use formula (18) for the phase. The next lemma, whose proof is given in Appendix B, shows that the phase for the generic trajectory is always bigger than the one for the critical trajectory.

Lemma 4.2. For any value of $\omega \neq \omega_{c}$, let $P_{\omega}(t)=\left(x_{\omega}(t), y_{\omega}(t)\right)$ be a fixed point on the corresponding trajectory for $t \in\left(0, \frac{\pi}{2 a}\right]$. Then if there exists a $\lambda$ as in Eq. (37) such that the absolute value of $P_{\omega}(t)$ is equal to the absolute value of the point of the critical trajectory corresponding to $\lambda$, then the phase of $P_{\omega}(t)$ is strictly bigger than the phase of this point. 
Corollary 4.3. The critical trajectory is entirely contained in the interior of $\mathcal{S}$ (except for the point $(1,0))$.

Proof. The separatrix is in the first and second quadrant only until time $t=\frac{\pi}{2 a^{*}}$ and therefore, using Lemma 4.2 and Proposition 4.1, intersection cannot occur (except for the point $(1,0)$ ).

Lemma 4.2 has two additional important consequences, which are presented in the next two Corollaries. The first one is that every trajectory corresponding to $\omega \in\left(\omega^{*},+\infty\right), \omega \neq \omega_{c}$ that intersects the critical trajectory has to do so at a time $t>\frac{\pi}{2 a}$. The second one is that all trajectories corresponding $\omega \in\left(\omega_{c},+\infty\right)$ do not reach any of the points below the critical curve and outside the critical circle (i.e., points in the region $\mathrm{R}$ of Figure 5), before hitting the boundary of the unit disk at time $t=\frac{\pi}{a}$.

Corollary 4.4. Any trajectory corresponding to a value of $\omega$ and $a$, with $\omega \neq \omega_{c}$ cannot intersect the critical trajectory for $t \leq \frac{\pi}{2 a}$.

Proof. Assume, by contradiction, that there exists an $\omega \neq \omega_{c}$ and a time $t \in\left(0, \frac{\pi}{2 a}\right]$, such that the corresponding trajectory intersect the critical trajectory. Denote by $P$, the point of intersection. Then, there exists a $\lambda \in(0,1]$ such that the absolute value of $P$ is equal to the absolute value of the point of the critical trajectory corresponding to $\lambda$. By applying Lemma 4.2, we know that the phase of $P$ is strictly bigger than the phase of this point. If they coincide, their phases must be equal up to a multiple of $2 \pi$. However, under the assumption $\gamma \leq \frac{1}{\sqrt{3}}$, the critical trajectory (until time $\frac{\pi}{2 a_{c}}$ ) is in the first quadrant while a trajectory corresponding to $\omega$ has phase which, until time $t=\frac{\pi}{2 a}$, can be calculated using (18) and we have

$$
0 \leq \text { phase of } P_{\omega} \leq \frac{\pi}{2}+\frac{\pi}{2}\left(\max _{\omega \in(-\infty, \infty)} \frac{\omega}{a}\right) \leq \frac{\pi}{2}+\pi .
$$

The last inequality is obtained by considering the function $f(\omega)=\frac{\omega}{a}$ which has its absolute maximum at $\omega=\omega_{c}$, which is equal to $f\left(\omega_{c}\right)=\frac{\sqrt{\gamma^{2}+1}}{\gamma}$. This, as a function of $\gamma$, attains its maximum value in the interval $\left[\frac{1}{\sqrt{3}}, 1\right]$ at the endpoint $\frac{1}{\sqrt{3}}$ and this value is equal to 2 .

From (38), it follows that the two points cannot coincide.

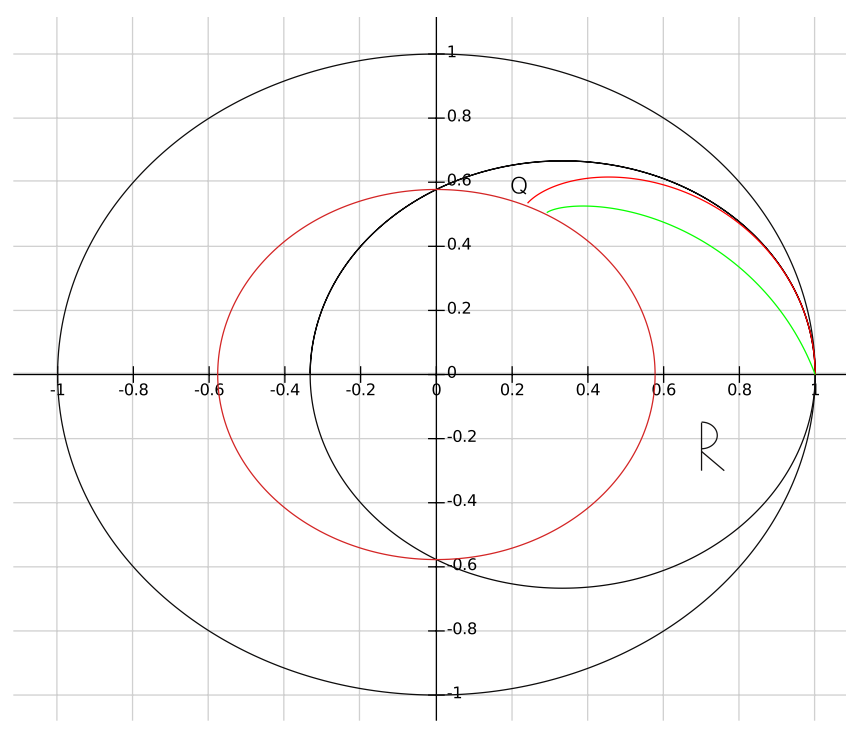

FIG. 5. Geometric objects used in the proof of Theorem 2 depicted here for the case $\gamma=\frac{1}{\sqrt{2}}$. The critical trajectory and the critical circle are in red. We deform the critical trajectory by adding a $-\epsilon \lambda$ to the phase. The corresponding deformed curve is in green. Trajectories for $\omega \in\left(\omega_{c}, 3 \omega^{*}\right]$ never reach the region $\mathrm{R}$ in the figure. 
Corollary 4.5. Consider a trajectory corresponding to $\omega \in\left(\omega_{c},+\infty\right)$. Such a trajectory never enters the critical circle. Moreover, if it goes in the region below the critical trajectory or intersect the critical trajectory, it does so after going outside the region $\mathcal{S}$.

The region below the critical trajectory is the region $R$ in Figure 5, i.e., the region outside the critical circle, inside the separatrix, and below the critical trajectory. The assumption $\gamma \geq \frac{1}{\sqrt{3}}$ guarantees that the critical circle is not completely included in the interior of $\mathcal{S}$. Recall that after going outside of $\mathcal{S}$ any trajectory cannot be optimal anymore since it intersects the optimal trajectories going to the boundary of the unit disk. This corollary says that optimal trajectories for points in $\mathrm{R}$ have to be sought for among the ones corresponding to values of $\omega \in\left[\omega^{*}, \omega_{c}\right]$.

Proof. Fix an $\omega \in\left(\omega_{c},+\infty\right)$, and let $P_{\omega}(t)$ be the point at time $t$ of the corresponding trajectory, and $P_{c}(t)$ the point at time $t$ of the critical trajectory. We first show that $P_{\omega}(t)$ never enters the critical circle. Since for $\omega>\omega_{c}>1, a=\sqrt{\gamma^{2}+(1-\omega)^{2}}$ is an increasing function of $\omega$, we have $a>a_{c}$ and $1-\frac{\gamma^{2}}{a^{2}}>1-\frac{\gamma^{2}}{a_{c}^{2}}$, which is the square of the radius of the critical circle. From formula (17), we have

$$
\left|P_{\omega}(t)\right|^{2}=1-\frac{\gamma^{2}}{a^{2}} \sin ^{2}(a t) \geq 1-\frac{\gamma^{2}}{a^{2}}>1-\frac{\gamma^{2}}{a_{c}^{2}},
$$

which proves the claim.

Now, we follow the trajectory $P_{\omega}(t)$. We know from Fact 1 in Subsection II C that the absolute value of $P_{\omega}(t)$ is bigger than the absolute value of $P_{c}(t)$ for $t$ in a suitable neighborhood of 0 . From Lemma 4.2, we also have that the phase of $P_{\omega}(t)$ is bigger than the one of $P_{c}(t)$. Thus, near $t=0$, the trajectory corresponding to $\omega$ is in the region above the critical curve; it either goes outside $\mathcal{S}$ or it stays in the region $Q$ of Figure 5.

Since it cannot enter the critical circle, in order to reach the region below the critical trajectory (region R of Figure 5) $P_{\omega}(t)$ can only either exit the region $\mathcal{S}$ or cross the critical trajectory. We want to show that the first case is the only one possible. For $t=\frac{\pi}{a}, P_{\omega}(t)$ is at the boundary of the unit disk, therefore outside $\mathcal{S}$. So, we only have to show that no intersection is possible for $t \in\left(0, \frac{\pi}{a}\right]$. In fact, since by Corollary 4.4 we know that $P_{\omega}(t)$ does not intersect the critical trajectory for $t \in\left(0, \frac{\pi}{2 a}\right]$, we only have to show that intersection cannot occur for $t \in\left(\frac{\pi}{2 a}, \frac{\pi}{a}\right)$, unless $P_{\omega}(t)$ exits the region $\mathcal{S}$.

Consider $P_{\omega}\left(\frac{\pi}{2 a}\right)$ and let $\bar{t} \in\left(0, \frac{\pi}{2 a_{c}}\right)$ be such that $\left|P_{\omega}\left(\frac{\pi}{2 a}\right)\right|=\left|P_{c}(\bar{t})\right|$. Let $\bar{\lambda}=\sin \left(a_{c} \bar{t}\right)$ (see Eq. (37)). Since for $t_{\omega} \in\left(\frac{\pi}{2 a}, \frac{\pi}{a}\right],\left|P_{\omega}\left(t_{\omega}\right)\right|>\left|P_{\omega}\left(\frac{\pi}{2 a}\right)\right|$ and for $t_{c} \in\left(\bar{t}, \frac{\pi}{2 a_{c}}\right],\left|P_{c}(\bar{t})\right|>\left|P_{c}\left(t_{c}\right)\right|$, we have

$$
\left|P_{\omega}\left(t_{\omega}\right)\right|>\left|P_{\omega}\left(\frac{\pi}{2 a}\right)\right|=\left|P_{c}(\bar{t})\right|>\left|P_{c}\left(t_{c}\right)\right|
$$

for all $t_{c} \in\left[\bar{t}, \frac{\pi}{2 a_{c}}\right]$. Therefore, the second part of the trajectory $P_{\omega}$ does not intersect the second part of the critical trajectory. It remains to show that it does not intersect the first part $(t<\bar{t})$ either.

The phase of $P_{\omega}\left(\frac{\pi}{2 a}\right)$ is bigger than the phase of $P_{c}(\bar{t})$ from Lemma 4.2, and so also bigger than the phase of $P_{c}(t)$ for $t \in[0, \bar{t}]$, since the phase is increasing from Fact 2, in Subsection II C. Moreover, for the same reason, the phase of $P_{\omega}(t)$, for $t \in\left(\frac{\pi}{2 a}, \frac{\pi}{a}\right)$ is bigger than the phase of $P_{\omega}\left(\frac{\pi}{2 a}\right)$, so, for $t \in\left(\frac{\pi}{2 a}, \frac{\pi}{a}\right)$, we have

$$
\text { phase of } P_{\omega}(t)>\text { phase of } P_{c}(\bar{t}) \geq \text { phase of } P_{c}\left(t_{c}\right) \quad t_{c} \in[0, \bar{t}] \text {. }
$$

This condition is compatible with intersection only if the phase of $P_{\omega}$ differs from the phase of $P_{c}$ by a multiple of $2 \pi$. Using Proposition 4.1 , in order for the intersection to occur, the trajectory $P_{\omega}$ should exit the region $\mathcal{S}$ through the separatrix.

Now we continuously deform the critical trajectory so that, for every $\lambda$, the new curve, which is still parametrized by $\lambda \in[0,1]$, is below the critical trajectory but still inside $\mathcal{S}$ and outside the critical circle (except (possibly) for the endpoints). Because of Proposition 4.1, we also deform it only slightly, so that the corresponding curve is entirely contained in the interior of the first quadrant (except for the point $(1,0)$ ). We call such a curve an $\epsilon$-curve. More precisely, an $\epsilon$-curve is obtained as follows. Choose a small $\epsilon>0$ with (at least)

$$
\epsilon<\min \left(1, \frac{1}{a_{c}^{2}}\right) .
$$


For every $\lambda \in[0,1]$, the corresponding point on the $\epsilon$-curve has the same radius as the corresponding point on the critical trajectory and phase $\psi_{\epsilon}(\lambda):=\psi(\lambda)-\epsilon \lambda$, where $\psi(\lambda)$ is the corresponding phase for the critical trajectory. Notice that for $\lambda=0$, the phases are the same (cf. Figure 5). Also choose $\epsilon$ small enough so that the $\epsilon$-curve is entirely contained in the first quadrant. ${ }^{35}$

We give two lemmas whose proofs are in Appendix B. They represent a crucial step in the proof of Theorem 2. They describe a map, $\zeta^{\epsilon}$, which associates to points on the $\epsilon$-curve the frequency $\omega$ giving the optimal control.

Lemma 4.6. Consider an $\epsilon$-curve with $\epsilon>0$ and satisfying (41), sufficiently small so that the curve is entirely contained in $\mathcal{S}$ and in the interior of the first quadrant (except for the point $(1,0)$ ). Let $\lambda_{\epsilon}:=\sqrt{1-\epsilon}$. Then there exists a function $\zeta^{\epsilon}:=\zeta^{\epsilon}(\lambda)$ with domain $\left[0, \lambda_{\epsilon}\right]$ and range $\left[\omega^{*}, \omega_{\epsilon}\right] \subset$ $\left[\omega^{*}, \omega_{c}\right)$, such that for $\lambda \in\left(0, \lambda_{\epsilon}\right), \zeta^{\epsilon}(\lambda)$ is the unique value $\omega$ which gives the optimal trajectory for the point on the $\epsilon$-curve corresponding to $\lambda . \zeta^{\epsilon}(0):=\omega^{*}, \zeta^{\epsilon}\left(\lambda_{\epsilon}\right):=\omega_{\epsilon}$. Furthermore, $\zeta^{\epsilon}$ satisfies the following properties.

1. $\zeta^{\epsilon}$ is a differentiable, increasing function from $\left[0, \lambda_{\epsilon}\right]$, one to one, and onto $\left[\omega^{*}, \omega_{\epsilon}\right] \subseteq\left[\omega^{*}, \omega_{c}\right)$.

2. For a given $\lambda$, the curve corresponding to $\zeta^{\epsilon}(\lambda)$, intersects the $\epsilon$-curve optimally at the first intersection and coming from below.

The idea of the proof is that since this $\epsilon$-curve is in the region below the critical curve and outside the critical circle all trajectories with $\omega \in\left(\omega_{c}, \infty\right)$ cannot reach it optimally (they touch the boundary of the unit disk first (Corollary 4.5)). Thus, the only trajectories left are the ones in $\left[\omega^{*}, \omega_{c}\right]$. So the map $\zeta^{\epsilon}$ maps to the right set. To construct the function $\zeta^{\epsilon}$ we fix a point $P$ on the $\epsilon$-curve. Then, $P$ is written both as a function of the parameter $\lambda$ and as a function of the control $\omega$. By equating the two expressions, in particular the absolute value and the phase up to a multiple of $2 k \pi$ of $P$, we find an implicit equation satisfied by the pair $(\lambda, \omega)$ which, by using the implicit mapping theorem, gives the desired function and has the properties described in the Lemma. Here, the assumption $\gamma \geq \frac{1}{\sqrt{3}}$ is used to show that only the case $k=-1$ has to be used in the multiple $2 k \pi$.

Lemma 4.7. Let $\omega_{\epsilon}$ be defined as in Lemma 4.6. For $\epsilon$ sufficiently small $\omega_{\epsilon}$ increases as $\epsilon$ decreases and we have

$$
\lim _{\epsilon \rightarrow 0^{+}} \omega_{\epsilon}=\omega_{c}
$$

\section{A. Proof of Theorem 2}

Proof of 1 . The proof is obtained by excluding optimality for values of $\omega \neq \omega_{c}$. If $\omega<\omega^{*}$, then all the trajectories loose their optimality after reaching the boundary and so before intersecting the critical trajectory, since until that point they are completely outside the separatrix. If $\omega=\omega^{*}$, then the trajectory is the separatrix which does not intersect the critical trajectory (except for the point $(1,0)$ ). Using Corollary 4.4 we know that any trajectory corresponding to a value of $\omega$ and $a$, with $\omega \neq \omega_{c}$ intersects the critical trajectory after $\frac{\pi}{2 a}$. Now we consider $\omega \in\left(\omega^{*}, \omega_{c}\right)$. The function $a$ of $\omega$ has a minimum at 1 , no local maximum in the interval $\left[\omega^{*}, \omega_{c}\right]$ and at the endpoints is given by $a^{*}=\frac{1+\gamma^{2}}{2}$ and $a_{c}=\gamma \sqrt{1+\gamma^{2}}$. Under the assumption $\gamma \geq \frac{1}{\sqrt{3}}, a_{c}$ is the largest of the two and therefore $a_{c}>a$ for every $\omega \in\left(\omega^{*}, \omega_{c}\right)$. Since this gives $\frac{\pi}{2 a}>\frac{\pi}{2 a_{c}}$ the intersection (if any) must happen, from Corollary 4.4, after $\frac{\pi}{2 a}$. However, this is the time to go through the whole critical trajectory, and therefore the trajectory corresponding to $\omega$ cannot be optimal. If $\omega>\omega_{c}$, using Corollary 4.5 , we have that the corresponding trajectory has to leave the region $\mathcal{S}$, and therefore looses optimality, before intersecting the critical trajectory. This leaves the only value $\omega=\omega_{c}$.

Proof of 2. Analogously to the previous case, the proof is obtained by excluding optimality for values of $\omega \neq \omega^{*}$.

If $\omega<\omega^{*}$, then all the trajectories may intersect the separatrix only after reaching the boundary, and so after having lost optimality (Lemma 3.3). Therefore, for any point on the separatrix, the optimal trajectory must correspond to a value of $\omega \in\left[\omega^{*},+\infty\right)$. The assumption $\gamma \geq \frac{1}{\sqrt{3}}$ guarantees 
that there are two (coinciding in the case $\gamma=\frac{1}{\sqrt{3}}$ ) points of intersection between the critical circle and the separatrix (cf. Figure 5). Consider the first of these two points. For all points that follow it on the separatrix the optimal control reaching these points cannot correspond to values of $\omega$ in the interval $\left(\omega_{c},+\infty\right)$, because of Corollary 4.5. ${ }^{36}$ Therefore, for these points, the optimal control has to correspond to a value $\omega \in\left[\omega^{*}, \omega_{c}\right]$. We now show that trajectories corresponding to $\omega \in\left(\omega^{*}, \omega_{c}\right)$ cannot be optimal for these points. Assume by contradiction that there exists an $\bar{\omega} \in\left(\omega^{*}, \omega_{c}\right)$ such that the corresponding trajectory is optimal for a point $P$ on the separatrix following the first point of intersection with the critical circle. From formula (42) of Lemma 4.7, we can choose $\epsilon$ sufficiently small so that $\bar{\omega}$ and therefore $\left(\omega^{*}, \bar{\omega}\right)$ is in the range of the function $\zeta^{\epsilon}$ of Lemma 4.6. Consider now the trajectory corresponding to $\bar{\omega}$ which by assumption is optimal for the point $P$. The point $P$ must occur before the intersection of the $\epsilon$-curve or after the trajectory is only tangent to the $\epsilon$-curve. ${ }^{37}$ The point $P$ cannot be after a crossing of the $\epsilon$-curve because after crossing the $\epsilon$-curve the trajectory will have to cross it again and-or cross itself to reach the point $P$, this way loosing optimality (cf. Lemma 4.6). ${ }^{38}$ Consider $\omega$, with $\omega^{*}<\omega<\bar{\omega}$ optimal for a point on the $\epsilon$-curve on the right of the point on the $\epsilon$-curve corresponding to $\bar{\omega}$. Such a trajectory starts above the trajectory for $\bar{\omega}$ and therefore it has to cross it (since the trajectory corresponding to $\bar{\omega}$ contains the point $P$ of the separatrix and both trajectories cannot leave the region $\mathcal{S}$, since otherwise they would loose optimality). This contradicts the fact that both of them are optimal, and gives the desired contradiction.

For the given points (after the first point of intersection), the trajectory corresponding to $\omega_{c}$ cannot be optimal either. This is easily seen because the trajectory is always in the interior of $\mathcal{S}$ until $t=\frac{\pi}{2 a_{c}}$. Moreover, by using the definitions, it is easy to check that $\gamma \leq 1$ implies $\frac{\pi}{2 a_{c}} \geq \frac{\pi}{a^{*}}$, and therefore for $t>\frac{\pi}{2 a_{c}}$ we already employ more time than the full separatrix curve.

In conclusion for the points on the separatrix after the first point of intersection with the critical circle, the only possibility for optimal trajectory is the separatrix itself. By the principle of optimality (Fact 4 of Subsection II C), it is optimal for the points before as well.

Proof of 3. If the point we consider is below the critical trajectory or inside the critical circle, then, by Corollary 4.5 , the optimal control frequency must be in $\left(\omega^{*}, \omega_{c}\right)$. Therefore, for these points, we only have to show that the corresponding $\omega$ is unique. Assume by contradiction that there are two values of $\omega$ leading to the same point $P$ optimally. Then, one of the two trajectories has to loose optimality at $P$. Let us denote by $\bar{\omega}$ the corresponding $\omega$. In Lemmas 4.6 and 4.7, we can use an $\epsilon$ sufficiently small so that $\bar{\omega}$ is in the range of the function $\zeta^{\epsilon}$. The point $P$ is either inside the critical circle or (if it is in the region $\mathrm{R}$ of Figure 5) below the $\epsilon$-curve. The intersection between the trajectory corresponding to $\bar{\omega}$ and $P$ has to occur before the intersection with the $\epsilon$-curve because otherwise the $\bar{\omega}$-trajectory will have to intersect the $\epsilon$-curve twice or intersect itself, thus loosing optimality (cf. Lemma 4.6). However, the loss of optimality at $P$ contradicts the optimality for the corresponding point on the $\epsilon$-curve.

By taking the limit as $\epsilon \rightarrow 0^{+}$and using Lemma 4.7 it follows that all trajectories corresponding to $\omega \in\left(\omega^{*}, \omega^{c}\right)$ loose optimality at the critical trajectory.

It remains to show that these are also the optimal trajectories for the points outside the critical circle and above the critical trajectory, i.e., points in the region $Q$ of Figure 5. The proof of this part follows similar lines as the proof of statement 2 in Proposition 3.4. In particular, consider $Q$ the region inside the separatrix, above the critical curve, and outside the critical circle (see Figure 5). Fix any point $P \in \mathrm{Q}$, we want to show that there exist a value $\bar{\omega} \in\left(\omega^{*}, \omega_{c}\right)$ such that the trajectory corresponding to this value passes through $P$ before loosing optimality, i.e., before intersecting the critical curve. Fix any curve $C=C(\tau)$ for $\tau \in[0,1]$, passing through $P$ such that $C(\tau) \in \mathrm{Q}$ if $\tau \in(0,1), C(0)=P_{0}$ is on the separatrix, and $C(1)=P_{1}$ is on the critical trajectory. Let $\bar{\tau}$ such that $C(\bar{\tau})=P$. Define $A^{-} \subset\left[\omega^{*}, \omega_{c}\right]$ (respectively, $A^{+} \subset\left[\omega^{*}, \omega_{c}\right]$ ) the set of $\omega$ 's such that $\omega \in A^{-}$(respectively, $\omega \in A^{+}$) if the trajectory corresponding to $\omega$ intersects, before loosing optimality (i.e., before intersecting the critical curve), the curve $C$ in a point $P_{\omega}=C(\tau)$ with $\tau \leq \bar{\tau}$ (respectively, $\tau \geq \bar{\tau}$ ). We have that $\omega^{*} \in A^{-}$, and $\omega_{c} \in A^{+}$, moreover, since any trajectory with $\omega \in\left(\omega^{*}, \omega_{c}\right)$ must intersects the curve $C$ before intersecting the critical curve, $A^{-} \cup A^{+}=\left[\omega^{*}, \omega_{c}\right]$. Using a continuity argument and since all $\omega$ 's are in a compact set, we have that both $A^{-}$and $A^{+}$are closed, thus, by connectedness, we must have that $A^{-} \cap A^{+} \neq \emptyset$. Necessarily if $\bar{\omega} \in A^{-} \cap A^{+}$, we have that its corresponding trajectory goes through $P$ before loosing optimality, as desired. 


\section{DISCUSSION}

The above analysis provides a description of the optimal trajectories for every element in $S U(2)$. It also gives a very simple method to find the optimal control for a fixed element $X_{f} \in S U(2)$.

Given such matrix one first singles out the $X_{1,1}$ element of the matrix and the point $P_{f}$ in the unit disk and checks whether $P_{f}$ is inside or outside the separatrix. If $P_{f}$ is outside, one has to use the trajectories described in Subsection III A, i.e., with $\omega \in\left(-\infty, \omega^{*}\right)$. The choice of $\omega$ can be made by successive approximations (for example, using a simple bisection algorithm) by examining the plots for trajectories which leave $P_{f}$ on the right or on the left and getting closer and closer to the trajectory which actually contains $P_{f}$. If $P_{f}$ is inside the separatrix, the same procedure can be performed with the trajectories described in Sec. IV. Once $\omega$ is found, one finds the corresponding $t$, either by tracing the plot or by solving an optimization problem minimizing (in $t$ ) the distance of the point on the trajectory from $P_{f}$. The last step is to adjust the phase $\tilde{\phi}$ in (14) (with the values found for $\omega$ and $t$ ) so that the element $X_{1,2}$ in (14) also coincides with the corresponding element in $X_{f}$. This completely determines the optimal controls in (13). If the mathematical problem is motivated by the time optimal steering of any quantum mechanical state $|\psi\rangle$ to $\pm X_{f}|\psi\rangle$, then the same procedure has to be repeated for the operator $-X_{f}$ to which it corresponds the point in the unit disk which is symmetric with respect to the origin. The control is chosen as the one which gives the minimum time between the two.

Figure 6 describes the work we have done to find the optimal control for the Hadamard gate $X_{f}:=\frac{1}{\sqrt{2}}\left(\begin{array}{cc}1 & 1 \\ -1 & 1\end{array}\right)$ and $\gamma=\frac{1}{\sqrt{2}}$. The point $P_{f}$ is the point $\left(\frac{1}{\sqrt{2}}, 0\right)$ which is inside the separatrix curve. We have drawn a small circle around this point. The two curves in blue in the figure correspond to $\omega=1.2 \omega^{*}, \omega=1.4 \omega^{*}$, and $\omega^{*}=\frac{3}{4}$ in this case. The optimal curve is found for $\omega:=\omega_{\text {opt }} \approx 1.28 \omega^{*}$ and is the red curve crossing the small circle in the figure. The optimal time is found to be approximately $t_{\text {opt }} \approx \pi+0.2$. The total phase of the $X_{1,2}$ element in (14) must be zero $(\sin (a t)$ is positive with the given values of $\omega=\omega_{\text {opt }}$ and $\left.t=t_{\text {opt }}\right)$, therefore, we choose $\tilde{\phi}=-\omega_{\text {opt }} t_{\text {opt }}=1.28 \omega^{*}(\pi+0.2)$. These values have to be replaced in (13) to give the optimal controls.

In a time optimal control problem for a two level quantum system, the work has to be repeated for $-X_{f}:=-\frac{1}{\sqrt{2}}\left(\begin{array}{cc}1 & 1 \\ -1 & 1\end{array}\right)$ where the corresponding point $-P_{f}=\left(-\frac{1}{\sqrt{2}}, 0\right)$ is the symmetric with respect to the origin of $P_{f}$. In this case, the point is outside the separatrix. Therefore, the optimal trajectory has to correspond to $\omega \in\left(-\infty, \frac{3}{4}\right)$. We have applied a bisection search starting from $\omega=0.5 \omega^{*}$ and $\omega=0.6 \omega^{*}$, and plotted the trajectories for $\omega=0.55 \omega^{*}$ and $\omega=0.575 \omega^{*}$. These trajectories are drawn

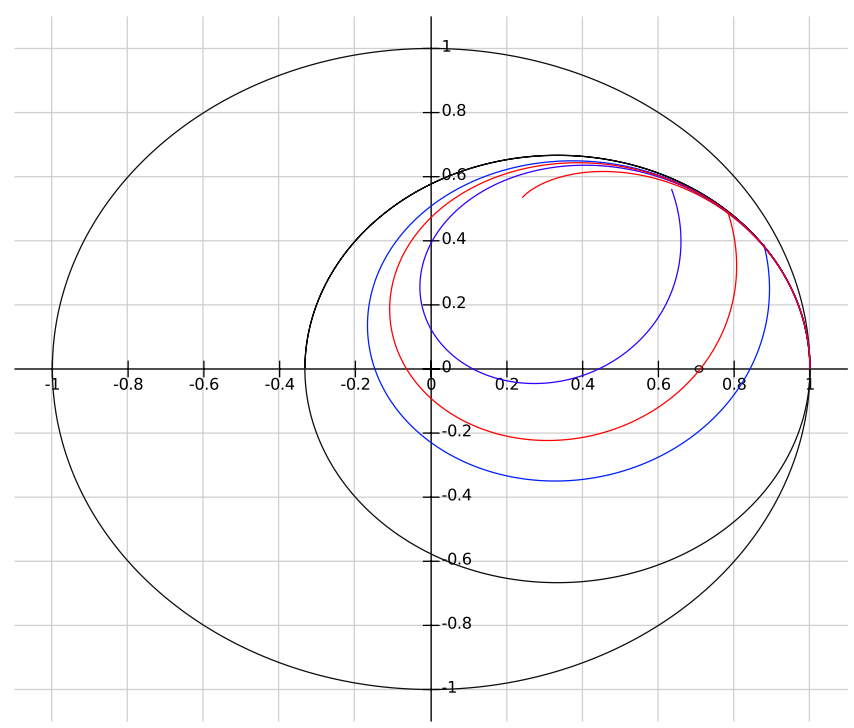

FIG. 6. Search for the optimal control for the Hadamard gate in the case $\gamma=\frac{1}{\sqrt{2}}$. The separatrix is in black and the critical trajectory is in red. Two trial trajectories corresponding to $\omega=1.2 \omega^{*}$ and $\omega=1.4 \omega^{*}$ are in blue and the (approximate) optimal trajectory is in red. 
in blue in Figure 7 except for the one of $\omega:=\omega_{\text {opt }}=0.575 \omega^{*}$ which is the optimal one as it crosses the desired point (and this optimal trajectory is in red). The optimal time is found to be approximately $t_{\text {opt }} \approx 0.7 \pi$, and therefore less than the time for $X_{f}$. Thus, this is the minimum time required for the optimal steering of a two level quantum system, and the frequency of the optimal control has to be chosen at $\omega_{\text {opt }}=0.575 \omega^{*}=0.575 \frac{3}{4}=0.43125$. Then we have also to choose the phase $\tilde{\phi}$ in (14) so that the total phase is equal to $\pi$ (because with the optimal values of $\gamma, \omega, a$, and $t, \frac{\gamma}{a} \sin (a t)=\frac{1}{\sqrt{2}}$ ). Therefore, we have $\tilde{\phi}=\pi-\omega_{\text {opt }} t_{\text {opt }}=\pi-\left(0.575 \omega^{*}\right) * 0.7 * \pi \approx 2.193$. We would like to stress that the knowledge of the optimal synthesis we have developed in the paper is crucial to obtain the solution of the practical problem of finding the minimum time control. Without the knowledge of the behavior of the optimal trajectories and using only the maximum principle and the reduction to the unit disk (Proposition 2.1), we only know that the optimal trajectories are parametrized by the frequency $\omega$. Then, given a point in the disk representing the (equivalence class of the) desired final condition, we should in principle select among the trajectories that cross that point (which all have in principle infinite length) the one that does it at the minimum time. This is a daunting task without knowing the qualitative behavior of the trajectories and where they loose optimality. This is what the optimal synthesis provides making the search for the optimal control possible.

While we were completing this work, other authors ${ }^{14}$ submitted a paper on the same topic, building upon their previous work in Ref. 13 for the case $\omega_{0}=0$ (cf. Remark 1.1) and previous work in Refs. 2 and 20. In the paper, ${ }^{14}$ the authors parametrize elements in $S U(2)$ with the so-called Hopf parameters ${ }^{39}$ and the Euler parameters of the elements of $S U(2)$. They derive the dynamical equations in terms of these parameters and consider the optimal control problem in this setting. They prove properties of the optimal trajectories and give an algorithm to find the optimal controls. Our geometric analysis of the optimal trajectories in the unit disk provides an alternative approach which, beside giving a very straightforward method to find the time optimal control, as we have seen above, highlights the general picture of the optimal trajectories. Main features of this picture are the existence of a closed curve which separates two classes of optimal trajectories (the separatrix) and of a special (non-smooth) trajectory inside this curve (the critical trajectory) which is some sort of limit of all other trajectories and it is where these trajectories loose optimality. It will be interesting in the future to investigate if, how and in what cases these features can be found in higher dimensional time optimal control systems on Lie groups.

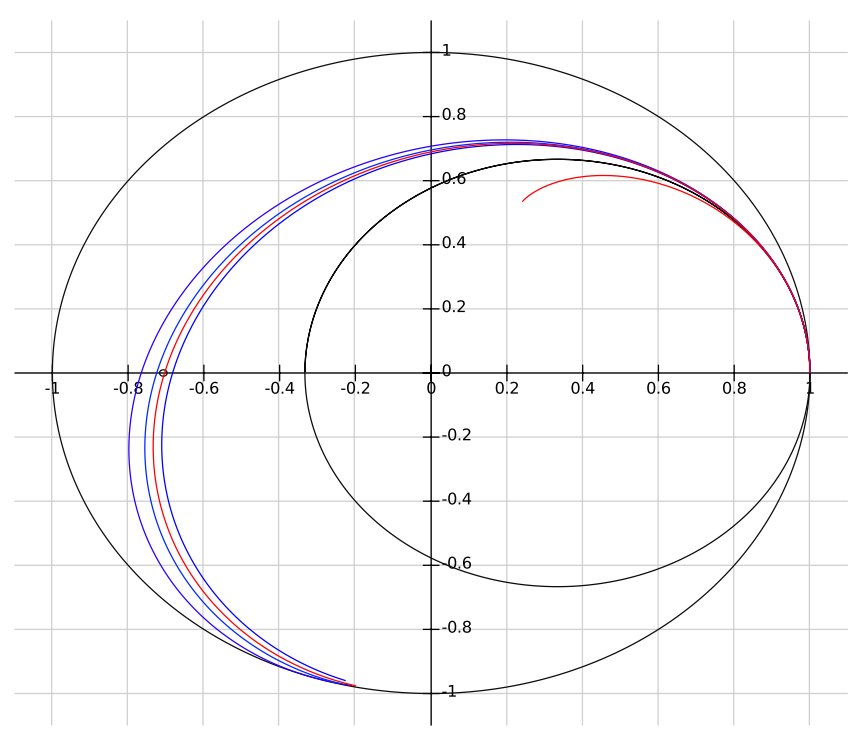

FIG. 7. Search for the optimal control for the opposite of the Hadamard gate, in the case $\gamma=\frac{1}{\sqrt{2}}$. The separatrix is in black and the critical trajectory is in red. Three trial trajectories corresponding to $\omega=0.6 \omega^{*}, \omega=0.5 \omega^{*}, \omega=0.55 \omega^{*}$ are in blue and the (approximate) optimal trajectory corresponding to $\omega=0.575 \omega^{*}$ is in red. 
While this paper was under review, another author, ${ }^{22}$ using the results described here, reconsidered the time optimal control on $S U(2)$ in the general case of unrestricted $\gamma$. He adopted our framework of analysis in the unit disk and introduced a novel ingredient, the evolution of the boundary of the reachable set, the "so called" front-line. It is a general fact that the geometry of the reachable sets is related to the time optimal control problem since if a point is reached optimally at time $T$ then it belongs to the boundary of the reachable set at the same time $T$ (see, e.g., Ref. 18). The paper ${ }^{22}$ visualizes the evolution of the reachable sets and their boundaries from which one can obtain the optimal control. We believe that this geometric description might be used to extend the analytical proof of the result of Theorem 2 removing the restriction on the value of $\gamma$. Overall, the paper ${ }^{22}$ is an important development of our work as it provides a general picture although with less detailed proofs which are visual in nature.

\section{ACKNOWLEDGMENTS}

D. D'Alessandro's research was partially supported by ARO MURI under Grant No. W911NF11-1-0268. D. D'Alessandro also acknowledges the kind hospitality of the Department of Mathematics at the University of Padova, Italy, where part of this work was performed. Graphs were drawn using "fooplot" at www.fooplot.com. The authors would like to thank Dr. D. Sugny for providing access to the paper. ${ }^{14}$

\section{APPENDIX A: SOME PROOFS OF RESULTS IN SECTION III}

\section{Proof of Lemma 3.1}

Proof. Using the expression of $a$ in terms of $\omega$, we have

$$
T_{k, m}=\frac{\pi k}{\sqrt{(1-\omega)^{2}+\gamma^{2}}}
$$

where $\omega$ is chosen to satisfy the Eq. (22), that is,

$$
1+\frac{\omega}{a}=1+\frac{\omega}{\sqrt{(1-\omega)^{2}+\gamma^{2}}}=\frac{\psi+2 m \pi}{k \pi} .
$$

Since the function $\frac{\omega}{a}$ has a maximum of $\frac{\sqrt{1+\gamma^{2}}}{\gamma}$ and an infimum at -1 , an $\omega \in \mathbf{R}$ satisfying (A2) exists if and only if

$$
0<\frac{\psi+2 m \pi}{k \pi} \leq 1+\frac{\sqrt{1+\gamma^{2}}}{\gamma}
$$

and there are at most two (solutions of second order algebraic equation) such $\omega$ 's.

From now on we assume to have fixed a value for $k$ as in the statement of the Lemma. Set also $\alpha(m):=\frac{\psi+2 m \pi}{k \pi}-1 \leq \frac{\sqrt{1+\gamma^{2}}}{\gamma}$. From (A1), $T_{k, m}$ is a decreasing function of $|b|:=|1-\omega|$. Therefore, the minimum $T_{k, m}$ will be obtained for the value of $m$ which corresponds to the maximum value of $|b|$, satisfying (A2), i.e.,

$$
f(b):=\frac{1-b}{\sqrt{b^{2}+\gamma^{2}}}=\alpha(m) .
$$

The claim of the Lemma is proved if we show that this is obtained for $m=0$.

It is easier to follow the proof if one looks at the shape of function $f$ in (A4) (see Figure 8). This function has its maximum in $b=-\gamma^{2}$, with $f\left(-\gamma^{2}\right)=\frac{\sqrt{\gamma^{2}+1}}{\gamma}$. Moreover, $f$ is strictly increasing for $b \in\left(-\infty,-\gamma^{2}\right)$, and strictly decreasing for $b \in\left(-\gamma^{2},+\infty\right)$. We have

$$
f\left(\left(-\infty,-\gamma^{2}\right)\right)=\left(1, \frac{\sqrt{\gamma^{2}+1}}{\gamma}\right) \quad f\left(\left(-\gamma^{2},+\infty\right)\right)=\left(-1, \frac{\sqrt{\gamma^{2}+1}}{\gamma}\right) .
$$




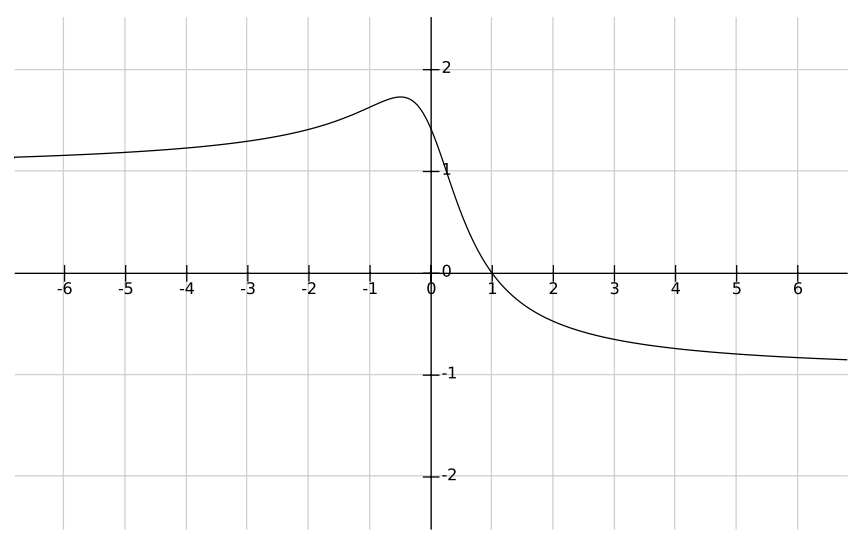

FIG. 8. Shape of the function $f$ defined in (A4). Here, the graph is given for $\gamma=\frac{1}{\sqrt{2}}$, but the properties used in the proof hold for any values of $\gamma$.

To every value of $m$, there will correspond a value $\alpha(m)$ and a horizontal line $(y=\alpha(m))$ in the graph. The value(s) of $b$ will be the one(s) where this line intersects the curve $y=f(b)$. We need to show that the value of $m$ which gives the $b$ with maximum absolute value is $m=0$.

It holds that

$$
-1<\alpha(0)=-1+\frac{\psi}{k \pi}<1,
$$

and that

$$
\alpha(m+1)=\alpha(m)+\frac{2 m}{k},
$$

from which it follows that $\alpha(m)$ is increasing with $m$. Consider first the values of $m \geq 0$ for which $\alpha(m) \leq 1$. For these values, there exists only one value of $b$ for which (A4) is verified. We denote this value by $b(m)$. Since $f(0)=\frac{1}{\gamma} \geq 1$ the value of $b(m)$ is greater than or equal to zero, and it is decreasing with $m$, i.e., $0 \leq b(m)<b(0)$.

The first $m$ such that $\alpha(m)>1$ is $m=k$, in which case $\alpha(m):=\alpha(k)=1+\frac{\psi}{k \pi}$. It is in fact easily seen that $\alpha(k-1)=-1+\frac{\psi}{k \pi}+2-\frac{2}{k}=1+\frac{\psi-2 \pi}{k}<1$.

For all $m>k$, since $\alpha(m)>1$, we have that either $\alpha(m)>\frac{\sqrt{\gamma^{2}+1}}{\gamma}$, then there are no solutions to the equation $f(b)=\alpha(m)$, or, if $\alpha(m) \leq \frac{\sqrt{\gamma^{2}+1}}{\gamma}$, then there are at most two solutions (indeed there are exactly two solutions for all values except in the case $\alpha(m)=\frac{\sqrt{\gamma^{2}+1}}{\gamma}$ where there is only one solution). Let us denote now by $b(m)$ the solution with the maximum absolute value. It is easy to show, by examining the solutions of the second order algebraic equation (A4) that $b(m)<0$, so we have that $b(m) \leq-\gamma^{2}$ and, since $f$ is increasing in $\left(-\infty,-\gamma^{2}\right)$ it holds that $|b(m)|<|b(k)|$.

Thus to show that $T_{k, m}$ is minimum at $m=0$, we need only to show that

$$
|b(k)|<b(0),
$$

where, again $b(k)$ now denotes the solution of $f(b)=\alpha(k)$ of maximum absolute value which is negative. This is equivalent to

$$
-b(k)<b(0) \text {. }
$$

Since the function $f(b)$ is decreasing for $b>0$, if we show that $f(-b(k))>f(b(0))$, then Eq. (A7) follows. We compute

$$
f(-b(k))=\frac{1+b(k)}{\sqrt{b(k)^{2}+\gamma^{2}}}=\frac{1-b(k)}{\sqrt{b(k)^{2}+\gamma^{2}}}+\frac{2 b(k)}{\sqrt{b(k)^{2}+\gamma^{2}}}=1+\frac{\psi}{k \pi}+\frac{2 b(k)}{\sqrt{b(k)^{2}+\gamma^{2}}} .
$$


Since $\left|\frac{b(k)}{\sqrt{b(k)^{2}+\gamma^{2}}}\right|<1$, we have that

$$
f(-b(k))>1+\frac{\psi}{k \pi}-2=f(b(0))
$$

as desired.

\section{Proof of Lemma 3.2}

Proof. For a given $\psi_{f} \in(0,2 \pi)$, the expression of $T_{k, 0}$ can be obtained from (21), where $\omega$ and $a=a(\omega)$ are chosen so that (22) is satisfied. In particular, after defining $x_{k, \psi}:=\frac{\psi_{f}-k \pi}{k \pi}$ and using the fact that $a$ is by definition positive, we obtain Ref. 40,

$$
a=\frac{-x_{k, \psi}+\sqrt{x_{k, \psi}^{2}+\left(1+\gamma^{2}\right)\left(1-x_{k, \psi}^{2}\right)}}{\left(1-x_{k, \psi}^{2}\right)} .
$$

Replacing this and the expression of $x_{k, \psi}$ in $T_{k, 0}=\frac{k \pi}{a}$, we obtain, after some algebra,

$$
T_{k, 0}=T_{k, 0}\left(\psi_{f}\right):=\frac{\psi_{f}\left(2 k \pi-\psi_{f}\right)}{k \pi-\psi_{f}+\sqrt{(k \pi)^{2}+\gamma^{2} \psi_{f}\left(2 k \pi-\psi_{f}\right)}} .
$$

We want to show that $T_{k, 0}\left(\psi_{f}\right)>T_{1,0}\left(\psi_{f}\right)$ for every $\psi_{f}$. Since $\psi_{f}>0$ is a multiplicative factor of very $T_{k, 0}$ we can neglect it in comparing the two functions. Moreover, since $\gamma$ is arbitrary, we can define $\gamma_{1}:=\gamma^{2} \psi_{f}>0$ and show, equivalently that $\tilde{T}_{k}\left(\psi_{f}\right)>\tilde{T}_{1}\left(\psi_{f}\right)$, for every $\psi_{f}$, with

$$
\tilde{T}_{k}\left(\psi_{f}\right):=\frac{2 k \pi-\psi_{f}}{k \pi-\psi_{f}+\sqrt{(k \pi)^{2}+\gamma\left(2 k \pi-\psi_{f}\right)}} .
$$

Write $Y_{k}:=(k \pi)^{2}+\gamma_{1}\left(2 k \pi-\psi_{f}\right)=Y_{1}+\Delta_{k}$, with $\Delta_{k}:=\left(k^{2}-1\right) \pi^{2}+2 \pi \gamma_{1}(k-1)$, so that the claim is equivalent to

$$
\left(2 k \pi-\psi_{f}\right)\left[\left(\pi-\psi_{f}\right)+\sqrt{Y_{1}}\right]>\left(2 \pi-\psi_{f}\right)\left[k \pi-\psi_{f}+\sqrt{Y_{1}+\Delta_{k}}\right] .
$$

After some algebra, we obtain

$$
\left(2 k \pi-\psi_{f}\right) \sqrt{Y_{1}}-(k-1) \pi \psi_{f}>\left(2 \pi-\psi_{f}\right) \sqrt{Y_{1}+\Delta_{k}} .
$$

Since both sides are positive, we can square both sides, and collecting the terms containing $Y_{1}$, and using the definition of $\Delta_{k}$, we arrive at

$$
\begin{gathered}
\left(4\left(k^{2}-1\right) \pi^{2}-4(k-1) \pi \psi_{f}\right) Y_{1}+(k-1)^{2} \pi^{2} \psi_{f}^{2}-2(k-1) \pi \psi_{f}\left(2 k \pi-\psi_{f}\right) \sqrt{Y_{1}}> \\
\left(4 \pi^{2}-4 \pi \psi_{f}+\psi_{f}^{2}\right)\left(\left(k^{2}-1\right) \pi^{2}+2 \gamma_{1} \pi(k-1)\right) .
\end{gathered}
$$

dividing everything by $\pi(k-1)$, we obtain

$$
4\left((k+1) \pi-\psi_{f}\right) Y_{1}+(k-1) \pi \psi_{f}^{2}-2 \psi_{f}\left(2 k \pi-\psi_{f}\right) \sqrt{Y_{1}}>\left(4 \pi^{2}-4 \pi \psi_{f}+\psi_{f}^{2}\right)\left((k+1) \pi+2 \gamma_{1}\right) .
$$

By collecting all terms that contain $Y_{1}$ on the left hand side and after some algebra, we obtain

$$
\left(4(k+1) \pi-4 \psi_{f}\right) Y_{1}-2 \psi_{f}\left(2 k \pi-\psi_{f}\right) \sqrt{Y_{1}}>2 \pi \psi_{f}^{2}+4 \pi^{2}(k+1)\left(\pi-\psi_{f}\right)+2 \gamma_{1}\left(2 \pi-\psi_{f}\right)^{2} .
$$

Using the expression for $Y_{1}$ (but not under the square root), we obtain, after some algebra,

$$
\left(2 k \pi-\psi_{f}\right) \gamma_{1}\left(2 \pi-\psi_{f}\right)+\pi \psi_{f}\left(2 k \pi-\psi_{f}\right)-\psi_{f}\left(2 k \pi-\psi_{f}\right) \sqrt{Y_{1}}>0,
$$

which allows us to simplify the factor $\left(2 k \pi-\psi_{f}\right)$, so that the theorem is verified if

$$
\gamma_{1}\left(2 \pi-\psi_{f}\right)+\pi \psi_{f}>\psi_{f} \sqrt{Y_{1}} .
$$


Taking the squares of both sides and reintroducing the expression of $Y_{1}$, we obtain, after some algebra,

$$
\gamma_{1}^{2}\left(2 \pi-\psi_{f}\right)^{2}+2 \pi \psi_{f} \gamma_{1}\left(2 \pi-\psi_{f}\right)>\psi_{f}^{2} \gamma_{1}\left(2 \pi-\psi_{f}\right)
$$

which after dividing by $\gamma_{1}\left(2 \pi-\psi_{f}\right)$, gives

$$
\gamma_{1}\left(2 \pi-\psi_{f}\right)+2 \pi \psi_{f}>\psi_{f}^{2}
$$

This is certainly true for $\psi_{f} \in(0,2 \pi)$ since $2 \pi \psi_{f}>\psi_{f}^{2}$, which completes the proof.

\section{Proof of Lemma 3.3}

Proof. The optimal trajectories reaching the boundary of the unit disk do not intersect (before the boundary) because the intersection would mean that one of them is not optimal. Denote by $P_{\omega}(\cdot):=$ $\left(x_{\omega}(\cdot), y_{\omega}(\cdot)\right)$ any of these trajectories, parametrized by $\omega$, with $-\infty<\omega<\omega^{*}:=\frac{1+\gamma^{2}}{2}$. The function $\omega$ as a function of $\psi_{f}(27)$ is an increasing function. This implies that if $\omega_{2}>\omega_{1}$ the curve $P_{\omega_{2}}$ starts below the curve $P_{\omega_{1}}$, otherwise, they would have to intersect.

Assume now by contradiction that the curve $P_{\omega_{1}}$ at time $\bar{t}$ intersects the separatrix in the point $P:=f\left(t^{*}, \omega^{*}\right)$. Consider now a curve $P_{\omega_{2}}$, with $\omega_{2}>\omega_{1}$, and let $d_{P}$ denote the distance of $P$ from the curve $P_{\omega_{2}}$. Since there is no intersection between $P_{\omega_{1}}$ and $P_{\omega_{2}}, d_{P}>0$. Moreover, for every $\omega>\omega_{2}$, the distance of the curve from $P$ is greater than $d_{P}$, otherwise there would be intersection of this curve with $P_{\omega_{2}}$. Consider now $P_{\omega}\left(t^{*}\right)$ and take the $\operatorname{limit}_{\lim _{\omega \rightarrow \omega^{*}}} P_{\omega}\left(t^{*}\right)$, which by continuity must be $P$. However, this contradicts the fact that the distance of any curve with $\omega>\omega_{2}$ from $P$ must be greater than $d_{P}>0$.

\section{APPENDIX B: SOME PROOFS OF RESULTS IN SECTION IV}

\section{Proof of Lemma 4.2}

Proof. Consider any two points, one on the curve $P_{\omega}$ and one on the critical trajectory with equal distance from the origin, and in particular let $\lambda$ denote the corresponding value of the parameter on the critical trajectory with $\lambda \in(0,1]$. Using formula (17), we have, for $t$ the corresponding time on the curve $P_{\omega}$,

$$
\frac{\sin ^{2}(a t)}{a^{2}}=\frac{\lambda^{2}}{a_{c}^{2}}
$$

From this equation assuming $t \leq \frac{\pi}{2 a}$, we derive

$$
\sin (a t)=\frac{a \lambda}{a_{c}}
$$

and therefore

$$
t=\frac{1}{a} \arcsin \left(\frac{a \lambda}{a_{c}}\right)
$$

Notice in particular that (B1) implies

$$
a_{c} \geq a \lambda
$$

From Eq. (B1), we get $\cos (a t)=\frac{\sqrt{a_{c}^{2}-a^{2} \lambda^{2}}}{a_{c}}$, and we also have

$$
\tan (a t)=\frac{a \lambda_{0}}{\sqrt{a_{c}^{2}-a^{2} \lambda^{2}}}
$$

if $a_{c}^{2}-a^{2} \lambda^{2}>0$ (cf. (B3)). Let $\Phi_{P}(\lambda)$ be the phase of a point on the curve $P_{\omega}$. By using Eq. (18) along with Eqs. (B2) and (B4), we have if $a_{c}^{2}-a^{2} \lambda^{2}>0$,

$$
\Phi_{P}(\lambda)=\omega \frac{1}{a} \arcsin \left(\frac{a \lambda}{a_{c}}\right)+\arctan \left(\frac{(1-\omega) \lambda}{\sqrt{a_{c}^{2}-a^{2} \lambda^{2}}}\right) .
$$


For the corresponding point on the critical trajectory, the phase $\Phi_{c}(\lambda)$ is obtained by the special case $\omega=\omega_{c}$ and $a=a_{c}$ (assuming in this case $\lambda<1$ ),

$$
\Phi_{c}(\lambda)=\omega_{c} \frac{1}{a_{c}} \arcsin (\lambda)+\arctan \left(\frac{\lambda\left(1-\omega_{c}\right)}{a_{c} \sqrt{1-\lambda^{2}}}\right) .
$$

We know that $\Phi_{P}(0)=\Phi_{c}(0)$, and we now fix a $\lambda_{0} \in(0,1]$. We want to prove that the phase corresponding to $\lambda_{0}$ for the trajectory $P_{\omega}$ is greater than the phase of the corresponding point on the critical trajectory. Since the two phases are the same for $\lambda=0$, it is enough to show that the derivative (with respect to $\lambda$ ) of the phase for $P_{\omega}$ is always greater than the derivative for the phase of the critical trajectory for every $\lambda<\lambda_{0} \leq 1$. Since Eq. (B3) holds for $\lambda=\lambda_{0}$, it holds with strict inequality for $\lambda<\lambda_{0}$, therefore $a^{2}-a_{c}^{2} \lambda^{2}>0$ and we can use (B4) and (B5) for the phase of the point on the trajectory $P_{\omega}$. Also, since $\lambda<\lambda_{0} \leq 1$, we can use formula (B6) for the phase of the point on the critical trajectory. Therefore, we will prove that $\Phi_{P}^{\prime}(\lambda)>\Phi_{c}^{\prime}(\lambda)$, for every $\lambda \in(0,1)$. We have

$$
\begin{gathered}
\Phi_{P}^{\prime}(\lambda)=\frac{\omega}{a} \frac{1}{\sqrt{\left(1-\frac{a^{2} \lambda^{2}}{a_{c}^{2}}\right)}} \frac{a}{a_{c}}+\frac{a_{c}^{2}-a^{2} \lambda^{2}}{a_{c}^{2}-a^{2} \lambda^{2}+(1-\omega)^{2} \lambda^{2}} \frac{(1-\omega)\left(a_{c}^{2}-a^{2} \lambda^{2}\right)+(1-\omega) a^{2} \lambda^{2}}{\left(a_{c}^{2}-a^{2} \lambda^{2}\right) \sqrt{a_{c}^{2}-a^{2} \lambda^{2}}}= \\
=\frac{\omega}{\sqrt{a_{c}^{2}-a^{2} \lambda^{2}}}+\frac{(1-\omega) a_{c}^{2}}{\left(a_{c}^{2}-\gamma^{2} \lambda^{2}\right) \sqrt{a_{c}^{2}-a^{2} \lambda^{2}}}=\frac{1}{\sqrt{a_{c}^{2}-a^{2} \lambda^{2}}} \frac{a_{c}^{2}-\omega \gamma^{2} \lambda^{2}}{a_{c}^{2}-\gamma^{2} \lambda^{2}} .
\end{gathered}
$$

Considering the special case $\omega=\omega_{c}$, and recalling the expression of $\omega_{c}$ and $a_{c}$ in terms of $\gamma$, we obtain

$$
\Phi_{c}^{\prime}(\lambda)=\frac{a_{c} \sqrt{1-\lambda^{2}}}{a_{c}^{2}-\gamma^{2} \lambda^{2}}
$$

Using Eqs. (B7) and (B8), we have

$$
\Phi_{P}^{\prime}(\lambda)>\Phi_{c}^{\prime}(\lambda) \Leftrightarrow \frac{a_{c}^{2}-\omega \gamma^{2} \lambda^{2}}{\sqrt{a_{c}^{2}-a^{2} \lambda^{2}}}>a_{c} \sqrt{1-\lambda^{2}} .
$$

Thus, we want to prove that

$$
a_{c}^{2}-\omega \gamma^{2} \lambda^{2}>a_{c} \sqrt{1-\lambda^{2}} \sqrt{a_{c}^{2}-a^{2} \lambda^{2}} .
$$

Now notice that the left hand side is always non-negative. In fact, we have

$$
a_{c}^{2}-\omega \gamma^{2} \lambda^{2} \geq a_{c}^{2}-\omega \gamma^{2}=\gamma^{2}\left(1-\omega+\gamma^{2}\right) \geq 0,
$$

where the last equality is verified if $\omega \leq \omega_{c}=1+\gamma^{2}$. Moreover, from formula (B3), we have

$$
a_{c}^{2}-\omega \gamma^{2} \lambda^{2} \geq a^{2} \lambda^{2}-\omega \gamma^{2} \lambda^{2}=(1-\omega) \lambda^{2}\left(1-\omega+\gamma^{2}\right) \geq 0,
$$

where the last inequality is verified if $\omega \geq 1+\gamma^{2}=\omega_{c}$. Therefore, to show (B9), it is sufficient to show the inequality on the squares of left and right hand side.

By taking the squares in (B9) we need to prove

$$
a_{c}^{4}+\omega^{2} \gamma^{4} \lambda^{4}-2 \omega \gamma^{2} \lambda^{2}-a_{c}^{2}\left(1-\lambda^{2}\right)\left(a_{c}^{2}-a^{2} \lambda^{2}\right)>0,
$$

which becomes

$$
\begin{gathered}
\lambda^{4}\left(\omega^{2} \gamma^{4}-a_{c}^{2} a^{2}\right)+\lambda^{2}\left(a_{c}^{2} a^{2}+a_{c}^{4}-2 \omega \gamma^{2} a_{c}^{2}\right)=\lambda^{2}\left(-\gamma^{2}\left(\omega-\omega_{c}\right)^{2} \lambda^{2}+a_{c}^{2}\left(\omega-\omega_{c}\right)^{2}\right)= \\
=\lambda^{2}\left(\omega-\omega_{c}\right)^{2}\left(a_{c}^{2}-\gamma^{2} \lambda^{2}\right)=\lambda^{2}\left(\omega-\omega_{c}\right)^{2} \gamma^{2}\left(\gamma^{2}+1-\lambda^{2}\right)>0,
\end{gathered}
$$

where the last equality holds since $\lambda<1$. 


\section{Proof of Lemma 4.6}

Proof. Given an $\epsilon$-curve, fix a value $\lambda \in\left(0, \lambda_{\epsilon}\right)$ and denote by $P^{\epsilon}(\lambda)$ the corresponding point on the $\epsilon$-curve and by $P_{c}(\lambda)$ the corresponding point on the critical trajectory. We have

$$
\left|P^{\epsilon}(\lambda)\right|=\left|P_{c}(\lambda)\right| \quad \text { and } \quad \psi_{\epsilon}(\lambda)=\psi(\lambda)-\epsilon \lambda,
$$

where $\psi_{\epsilon}(\lambda)$ denotes the phase of $P^{\epsilon}(\lambda)$ and $\psi(\lambda)$ denotes the phase of $P_{c}(\lambda)$. Since $P^{\epsilon}(\lambda)$ is in the region below the critical curve and in the interior of $\mathcal{S}$, we know by Corollary 4.5 , that the value(s) of $\omega$ that give the optimal control for the point $P^{\epsilon}(\lambda)$ must be in the interval $\left(\omega^{*}, \omega_{c}\right] .^{41}$ This gives a function from $\zeta^{\epsilon}:\left(0, \lambda_{\epsilon}\right) \rightarrow\left(\omega^{*}, \omega_{c}\right]$, if we know that $\omega$ is unique.

To prove uniqueness of the optimal value $\omega$, and to be able to construct the function $\zeta^{\epsilon}$, we first need to find some relations among the values of $\lambda, \omega$, and $t$, where $\omega$ is one of the optimal values of the frequency (in principle not unique at this point) and $t$ is the corresponding minimum time.

If $P_{\omega}(t)=P^{\epsilon}(\lambda)$, on an optimal trajectory, we know from Corollary 4.4 that $t$ must be larger than $\frac{\pi}{2 a}$ and it must be smaller than $\frac{\pi}{a} \cdot{ }^{42}$ Since the point $P^{\epsilon}(\lambda)$ has the same distance from the origin as the corresponding point on the critical trajectory, we have

$$
\left|P^{\epsilon}(\lambda)\right|^{2}=1-\frac{\lambda^{2}}{a_{c}^{2}}
$$

From (17),

$$
\left|P_{\omega}(t)\right|^{2}=1-\frac{\sin ^{2}(a t)}{a^{2}}
$$

and, from these two equations, since all quantities are positive, we have

$$
\frac{\sin (a t)}{a}=\frac{\lambda}{a_{c}} .
$$

Equation (B13) gives a first relation between $\lambda$ and $\omega$ and $t$. Next, by equating the phases of the two points $P^{\epsilon}(\lambda)$ and $P_{\omega}(t)$ we will find another relation which will enable us to eliminate the $t$ dependence and find an implicit formula of the type $F^{\epsilon}(\lambda, \omega)=0$. From this relation and using the implicit mapping theorem, we will prove our statements.

Using the definition of $\lambda$ given by Eq. (37), the expression of the phase given in Eq. (18), and the fact that the critical trajectory is in the first quadrant, we have

$$
\psi(\lambda)=\omega_{c} \frac{\arcsin (\lambda)}{a_{c}}+\arctan \left(\frac{\left(1-\omega_{c}\right) \lambda}{a_{c} \sqrt{1-\lambda^{2}}}\right) .
$$

Now, using Eq. (19), since $t>\frac{\pi}{2 a}$, we also have

$$
\text { Phase } P_{\omega}(t)=\omega t+\pi+\arctan \left(\frac{(1-\omega)}{a} \tan (a t)\right) .
$$

From Eq. (B13), and since $t \in\left(\frac{\pi}{2 a}, \frac{\pi}{a}\right)$ we have

$$
t=\frac{1}{a}\left(\pi-\arcsin \left(\frac{a \lambda}{a_{c}}\right)\right) .
$$

Furthermore, using again the fact that $t \in\left(\frac{\pi}{2 a}, \frac{\pi}{a}\right)$, we have from (B13), $\cos (a t)=-\sqrt{1-\frac{a^{2} \lambda^{2}}{a_{c}^{2}}}$, and therefore $\tan (a t)=-\frac{a \lambda}{\sqrt{a_{c}^{2}-a^{2} \lambda^{2}}}$.

Thus, using (B16), the previous equality, and Eq. (B13), we can rewrite Eq. (B15) as

$$
\text { Phase } P_{\omega}(t)=\frac{\omega}{a}\left(\pi-\arcsin \left(\frac{a \lambda}{a_{c}}\right)\right)+\pi+\arctan \left(-\frac{(1-\omega) \lambda}{\sqrt{a_{c}^{2}-\left(a^{2} \lambda^{2}\right)}}\right) \text {. }
$$

Since $P^{\epsilon}(\lambda)=P_{\omega}(t)$, the phases must be equal up to a multiple of $2 \pi$. Thus, we must have

$$
\psi_{\epsilon}(\lambda)=\text { Phase } P_{\omega}(t)+2 k \pi,
$$


for some $k \in \mathbb{Z}$. Since the $\epsilon$-curve is in the first quadrant, we have

$$
0 \leq \psi_{\epsilon}(\lambda) \leq \frac{\pi}{2}
$$

Since $\gamma \geq \frac{1}{\sqrt{3}}$, we have that $1 \leq \frac{\omega}{a} \leq 2$, and since the argument inside the function $\arcsin$ in Eq. (B17) is positive, we have ${ }^{43}$

$$
\frac{3 \pi}{2} \leq \text { Phase } P_{\omega}(t) \leq \frac{7}{2} \pi .
$$

Given the previous bound for the two phases, the only possible $k \in \mathbb{Z}$ for which equality (B18) holds is $k=-1$. Thus, we can rewrite equality (B18), using $k=-1$ and Eqs. (B14) and (B17). We have

$$
\begin{aligned}
& \frac{\omega}{a}\left(\pi-\arcsin \left(\frac{a \lambda}{a_{c}}\right)\right)+\pi+\arctan \left(-\frac{(1-\omega) \lambda}{\sqrt{a_{c}^{2}-a^{2} \lambda^{2}}}\right)-2 \pi= \\
& =\omega_{c} \frac{\arcsin (\lambda)}{a_{c}}+\arctan \left(\frac{\left(1-\omega_{c}\right) \lambda}{a_{c} \sqrt{1-\lambda^{2}}}\right)-\epsilon \lambda .
\end{aligned}
$$

For $\omega \in\left(\omega^{*}, \omega_{c}\right)$ (and recalling $\left.a=\sqrt{\left(\gamma^{2}+(1-\omega)^{2}\right)}\right)$ and $\lambda \in\left(0, \lambda_{\epsilon}\right)$, let ${ }^{44}$

$$
\begin{aligned}
F^{\epsilon}(\lambda, \omega)= & \frac{\omega}{a}\left(\pi-\arcsin \left(\frac{a \lambda}{a_{c}}\right)\right)-\arctan \left(\frac{(1-\omega) \lambda}{\sqrt{a_{c}^{2}-a^{2} \lambda^{2}}}\right)-\pi- \\
& \frac{\sqrt{\gamma^{2}+1}}{\gamma} \arcsin (\lambda)+\arctan \left(\frac{\gamma \lambda}{\sqrt{\left(\gamma^{2}+1\right)} \sqrt{\left(1-\lambda^{2}\right)}}\right)+\epsilon \lambda .
\end{aligned}
$$

Notice that this function is well defined for $\lambda \in\left(0, \lambda_{\epsilon}\right)$, since $a_{c}>a$ for $\omega \in\left(\omega^{*}, \omega_{c}\right)$ under the assumption $\gamma \geq \frac{1}{\sqrt{3}}$ (cf. the proof of part one of Theorem 2 in the main body of the paper). For any $\lambda \in\left(0, \lambda_{\epsilon}\right)$, and corresponding optimal $\omega$, Eq. (B19) says

$$
F^{\epsilon}(\lambda, \omega)=0 .
$$

Now we want to show that this gives a well defined function $\zeta^{\epsilon}(\lambda)=\omega$, i.e., that given $\lambda$ there is only one $\omega$ giving the optimal control for that $\lambda$ (uniqueness).

Uniqueness Assume by contradiction that there are two optimal $\omega$ 's for the same point on the $\epsilon$-curve. Both values of $\omega$ and corresponding $a$ 's must satisfy Eq. (B16), with the same $t$ (because of optimality). Since the right hand side of (B16) is a decreasing function of $a$, independently of $\lambda$, the two $a$ 's must coincide. This means that the two values of $\omega$, say $\omega_{1}$ and $\omega_{2}$, have to be such that $\left|1-\omega_{1}\right|=\left|1-\omega_{2}\right|$. Now recall that the function $a=a(\omega)$ has a minimum at $\omega=1$ and is symmetric around this point. Under the assumption $\gamma \geq \frac{1}{\sqrt{3}}$, the largest interval symmetric around $\omega=1$ and contained in $\left(\omega^{*}, \omega_{c}\right)$ is $\left(\omega^{*}, 2-\omega^{*}\right)$. The two values $\omega_{1}$ and $\omega_{2}$ must be symmetric in this interval. Therefore, we write $\omega_{1}:=\omega \in\left(\omega^{*}, 1\right), \omega_{2}:=2-\omega$. Both $\omega_{1}$ and $\omega_{2}$ must satisfy Eq. (B21), with the same $a$ and $\lambda$. Therefore, writing this equation for $\omega$ and for $\omega$ replaced by $2-\omega$, we obtain

$$
\begin{gathered}
\frac{\omega}{a}\left(\pi-\arcsin \left(\frac{a \lambda}{a_{c}}\right)\right)-\arctan \left(\frac{(1-\omega) \lambda}{\sqrt{a_{c}^{2}-a^{2} \lambda^{2}}}\right)= \\
\frac{2-\omega}{a}\left(\pi-\arcsin \left(\frac{a \lambda}{a_{c}}\right)\right)+\arctan \left(\frac{(1-\omega) \lambda}{\sqrt{a_{c}^{2}-a^{2} \lambda^{2}}}\right),
\end{gathered}
$$

which, rearranging the terms and dividing by 2 , gives

$$
\frac{\omega-1}{a}\left(\pi-\arcsin \left(\frac{a \lambda}{a_{c}}\right)\right)=\arctan \left(\frac{(1-\omega) \lambda}{\sqrt{a_{c}^{2}-a^{2} \lambda^{2}}}\right) .
$$

The left and right hand side have opposite signs and can coincide only when $\omega=1=2-\omega$. This shows the uniqueness. 
So, we can define

$$
\begin{aligned}
\zeta^{\epsilon}:\left(0, \lambda_{\epsilon}\right) & \rightarrow\left(\omega^{*}, \omega_{c}\right) \\
\lambda & \mapsto \zeta^{\epsilon}(\lambda)
\end{aligned}
$$

where $\zeta^{\epsilon}(\lambda)$ is the optimal value to reach the point $P^{\epsilon}(\lambda)$, and we know that

$$
F^{\epsilon}\left(\lambda, \zeta^{\epsilon}(\lambda)\right)=0 .
$$

Now, we use the implicit mapping theorem to prove all the properties (of differentiability and monotonicity) of the statement of the Lemma, and to extend the domain of the map at the values $\lambda=0$ and $\lambda=\lambda_{e}$. We call this part of the proof "Properties."

Properties We calculate $\frac{\partial F^{\epsilon}}{\partial \lambda}$ and $\frac{\partial F^{\epsilon}}{\partial \omega}$. We have from (B20)

$$
\begin{gathered}
\frac{\partial F^{\epsilon}}{\partial \lambda}(\lambda, \omega)=\frac{-\omega}{\sqrt{\left(a_{c}^{2}-a^{2} \lambda^{2}\right)}}-\frac{(1-\omega) a_{c}^{2}}{\gamma^{2}\left(\left(\gamma^{2}+1\right)-\lambda^{2}\right) \sqrt{\left(a_{c}^{2}-a^{2} \lambda^{2}\right)}}+ \\
-\frac{\sqrt{\left(\gamma^{2}+1\right)}}{\gamma} \frac{1}{\sqrt{1-\lambda^{2}}}+\frac{\gamma \sqrt{\left(\gamma^{2}+1\right)}}{\sqrt{1-\lambda^{2}}\left(\left(\gamma^{2}+1\right)-\lambda^{2}\right)}+\epsilon .
\end{gathered}
$$

Thus, with $\lambda \in\left(0, \lambda_{\epsilon}\right)$,

$$
\frac{\partial F^{\epsilon}}{\partial \lambda}(\lambda, \omega)=\frac{-\left(\gamma^{2}+1-\omega \lambda^{2}\right)}{\left(\gamma^{2}+1-\lambda^{2}\right) \sqrt{\left(a_{c}^{2}-a^{2} \lambda^{2}\right)}}-\frac{\sqrt{\gamma^{2}+1} \sqrt{1-\lambda^{2}}}{\gamma\left(\gamma^{2}+1-\lambda^{2}\right)}+\epsilon .
$$

Since, from (41), $\epsilon<\frac{1}{a_{c}^{2}}=\frac{1}{\gamma^{2}+\gamma^{4}}$ and $\lambda \in\left(0, \lambda_{\epsilon}\right)$, with $\lambda_{\epsilon}:=\sqrt{1-\epsilon}$ it holds that

$$
-\frac{\sqrt{\gamma^{2}+1} \sqrt{1-\lambda^{2}}}{\gamma\left(\gamma^{2}+1-\lambda^{2}\right)}+\epsilon<-\frac{\sqrt{\gamma^{2}+1} \sqrt{\epsilon}}{\gamma\left(\gamma^{2}+1-\lambda^{2}\right)}+\epsilon<-\frac{\sqrt{\epsilon}}{\gamma \sqrt{\gamma^{2}+1}}+\epsilon<0 .
$$

Thus, we have that

$$
\frac{\partial F^{\epsilon}}{\partial \lambda}(\lambda, \omega)<0
$$

We also have

$$
\frac{\partial F^{\epsilon}}{\partial \omega}(\lambda, \omega)=\frac{\gamma^{2}+1-\omega}{a^{3}}\left(\pi-\arcsin \left(\frac{a \lambda}{a_{c}}\right)\right)+\frac{\omega}{a^{2}} \frac{(1-\omega) \lambda}{\sqrt{a_{c}^{2}-a^{2} \lambda^{2}}}-\frac{\lambda}{\sqrt{a_{c}^{2}-a^{2} \lambda^{2}}} .
$$

Thus,

$$
\frac{\partial F^{\epsilon}}{\partial \omega}(\lambda, \omega)=\frac{\gamma^{2}+1-\omega}{a^{3}}\left(\pi-\arcsin \left(\frac{a \lambda}{a_{c}}\right)\right)+\frac{\lambda\left(\gamma^{2}+1-\omega\right)}{a^{2} \sqrt{a_{c}^{2}-a^{2} \lambda^{2}}} .
$$

From (B27) it follows that, for any $\lambda \in\left(0, \lambda_{\epsilon}\right)$ and $\omega \in\left(\omega^{*}, \omega_{c}\right)$, we have that

$$
\frac{\partial F^{\epsilon}}{\partial \omega}(\lambda, \omega)>0 .
$$

This from the implicit mapping theorem implies that $\zeta^{\epsilon}$ is continuously differentiable with derivative

$$
\frac{d \zeta^{\epsilon}}{d \lambda}=-\frac{\frac{\partial F^{\epsilon}}{\partial \lambda}(\lambda, \omega)}{\frac{\partial F^{\epsilon}}{\partial \omega}(\lambda, \omega)}>0
$$

where the inequality follows using Eq. (B26). So, for $\lambda \in\left(0, \lambda_{\epsilon}\right), \zeta^{\epsilon}$ is strictly increasing, and therefore one to one and onto an open interval in $\left(\omega^{*}, \omega_{c}\right)$.

Let $\omega_{\epsilon}=\sup _{\lambda \in\left(0, \lambda_{\epsilon}\right)} \zeta^{\epsilon}(\lambda)$ and define $\zeta^{\epsilon}\left(\lambda_{\epsilon}\right):=\omega_{\epsilon}$. Then, $\zeta^{\epsilon}$ is continuous at $\lambda=\lambda_{\epsilon}$. Define $\zeta^{\epsilon}(0)$ $:=\omega^{*}$, to prove continuity also at this point we need to show

$$
\lim _{\lambda \rightarrow 0^{+}} \zeta^{\epsilon}(\lambda)=\omega^{*}
$$

By monotonicity, we know that this limit exists. 
Let $\lim _{\lambda \rightarrow 0^{+}} \zeta^{\epsilon}(\lambda)=\bar{\omega}$, then since $F^{\epsilon}\left(\lambda, \zeta^{\epsilon}(\lambda)\right)=0$, and $F^{\epsilon}$ is continuous we must have $F^{\epsilon}(0, \bar{\omega})=$ 0 . It holds

$$
F^{\epsilon}(0, \bar{\omega})=\left(\frac{\bar{\omega}}{\bar{a}}-1\right) \pi,
$$

and this expression is zero if and only if $\frac{\bar{\omega}}{\bar{a}}=1$ which is equivalent to $\bar{\omega}=\omega^{*}$.

To complete the proof of the Lemma, we need to show the point 2 of its statement. First, we show that a trajectory that intersect the $\epsilon$-curve optimally does so at the first intersection. Assume by contradiction that the optimal intersection for a trajectory $\omega_{1}$ happens after the first intersection and denote by $P$ the point of the first intersection. At this point, there must exist another trajectory $\omega_{2}$ which reaches it optimally. However, this contradicts the fact that the $\omega_{1}$ trajectory intersect the $\epsilon$-curve optimally at a later time. ${ }^{45}$ To show that the optimal trajectory comes from below, using formula (20) at the point where the intersection occurs we show that the phase of the trajectory $P_{\omega}$ is increasing. At that time $t$ we have that Eq. (B13) is verified, so that, replacing the expression of $\sin ^{2}(a t)$ in (20), we obtain for the numerator (recalling the expressions of $\omega_{c}$ and $a_{c}$ )

$$
a^{2}\left(1-\gamma^{2} \frac{\lambda \omega}{a_{c}^{2}}\right)>a^{2}\left(1-\frac{\gamma^{2} \lambda^{2} \omega_{c}}{a_{c}^{2}}\right)=a^{2}\left(1-\lambda^{2}\right)>0 .
$$

\section{Proof of Lemma 4.7}

Proof. By definition and continuity we have, with the notations of Lemma 4.6,

$$
F^{\epsilon}\left(\lambda_{\epsilon}, \omega_{\epsilon}\right)=\lim _{\lambda \rightarrow \lambda_{\epsilon}} F^{\epsilon}\left(\lambda, \zeta^{\epsilon}(\lambda)\right)=0 .
$$

This equation defines $\omega_{\epsilon}$ implicitly as a function of $\epsilon$. In fact, if we calculate $\frac{\partial F^{\epsilon}}{\partial \omega_{\epsilon}}$ this is the same as in Eq. (B27) with $\lambda$ replaced by $\lambda_{\epsilon}$ and $\omega$ replaced by $\omega_{\epsilon}$ and therefore strictly positive (cf. Eq. (B28)). To calculate $\frac{\partial F^{\epsilon}}{\partial \epsilon}$, we see from (B20) that $F^{\epsilon}$ depends on $\epsilon$ directly and through $\lambda_{\epsilon}$. Therefore, we have from (B20)

$$
\frac{\partial F^{\epsilon}}{\partial \epsilon}=\left.\frac{\partial F^{\epsilon}}{\partial \lambda}\right|_{\lambda=\lambda_{\epsilon}} \frac{d \lambda_{\epsilon}}{d \epsilon}+\lambda_{\epsilon}
$$

Since $\left.\frac{\partial F^{\epsilon}}{\partial \lambda}\right|_{\lambda=\lambda_{\epsilon}}<0$ (cf. (B26)) and $\frac{d \lambda_{\epsilon}}{d \epsilon}=\frac{-1}{2 \sqrt{1-\epsilon}}<0$, we have that $\frac{\partial F^{\epsilon}}{\partial \epsilon}>0$, so that

$$
\frac{d \omega_{\epsilon}}{d \epsilon}=-\frac{\frac{\partial F}{\partial \epsilon}}{\frac{\partial F \epsilon}{\partial \omega_{\epsilon}}}<0
$$

which shows that $\omega_{\epsilon}$ is a decreasing function of $\epsilon$.

It remains to prove that $\lim _{\epsilon \rightarrow 0^{+}} \omega_{\epsilon}=\omega_{c}$. Let us denote by $\omega_{L}=\lim _{\epsilon \rightarrow 0^{+}} \omega_{\epsilon}$ and we have because of monotonicity and, boundedness for every $\epsilon$, that the limit $\omega_{L}$ exists and $\omega_{L} \leq \omega_{c}$. Moreover, by continuity, $\omega_{L}$ has to satisfy

$$
\lim _{\epsilon \rightarrow 0} F^{0}\left(\lambda_{\epsilon}, \omega_{L}\right)=0 .
$$

This, using (B20), gives that $\omega_{L}$ is the $\omega$ satisfying

$$
G(\omega)=\frac{\omega}{a}\left(\pi-\arcsin \left(\frac{a}{a_{c}}\right)\right)-\arctan \left(\frac{1-\omega}{\sqrt{a_{c}^{2}-a^{2}}}\right)-\frac{\pi}{2}+\frac{\sqrt{1+\gamma^{2}}}{\gamma} \frac{\pi}{2}=0 .
$$

Calculating $\frac{d G}{d \omega}$ we find that $\frac{d G}{d \omega}>0$ for every $\omega \in\left[\omega^{*}, \omega_{c}\right.$ ). Therefore, the Eq. (B34) has at most one solution in the interval $\left[\omega^{*}, \omega_{c}\right]$. By replacing $\omega_{c}$ in $(\mathrm{B} 34)$, one directly verifies that $\omega_{c}$ satisfies it. Therefore, $\omega_{L}=\omega_{c}$.

${ }^{1}$ C. Altafini, A. M. Bloch, and P. Rouchon, “Guest editorial: Special issue on control of quantum mechanical systems," IEEE Trans. Autom. Control 57(8), 1893 (2012).

2 A. D. Boozer, "Time optimal synthesis of $S U(2)$ transformations for a spin $\frac{1}{2}$ system," Phys. Rev. A 85, 012317 (2012). 
${ }^{3}$ U. Boscain, T. Chambrion, and J.-P. Gauthier, "On the K+P problem for a three-level quantum system: Optimality implies resonance,” J. Dyn. Control Syst. 8, 547-572 (2002).

${ }^{4}$ U. Boscain, F. Grönberg, R. Long, and H. Rabitz, "Time minimal trajectories for two level quantum systems with two bounded controls,” J. Math. Phys. 55, 062106 (2014).

${ }^{5}$ U. Boscain and P. Mason, "Time minimal trajectories for a spin $\frac{1}{2}$ particle in a magnetic field," J. Math. Phys. 47, 062101 (2006).

${ }^{6}$ C. Cohen-Tannoudji, B. Diu, and F. Laloe, Quantum Mechanics (Wiley, New York, 1977).

${ }^{7}$ D. D'Alessandro, Introduction to Quantum Control and Dynamics (CRC Press, Boca Raton, FL, 2007).

${ }^{8}$ H.-A. Engels, L. P. Kouwenhoven, D. Loss, and C. M. Markus, "Controlling spin qubits in quantum dots," Quantum Inf. Process. 3, 115 (2004).

${ }^{9}$ A. Carlini, A. Hosoya, T. Koike, and Y. Okudaira, "Time optimal quantum evolution," Phys. Rev. Lett. 96, 060503 (2006).

${ }^{10}$ A. Carlini, A. Hosoya, T. Koike, and Y. Okudaira, “Time optimal unitary evolutions," Phys. Rev. A 75, 042308 (2007).

${ }^{11}$ R. R. Ernst, G. Bodenhausen, and A. Wokaun, Principles of Nuclear Magnetic Resonance in One and Two Dimensions (Clarendon Press, Oxford, 1987).

12 W. Fleming and R. Rishel, Deterministic and Stochastic Optimal Control, Applications of Mathematics (Springer-Verlag, New York, 1975).

${ }^{13}$ A. Garon, S. J. Glaser, and D. Sugny, "Time-optimal control of SU(2) quantum operations," Phys. Rev. A 88, 043422 (2013).

14 A. Garon, S. J. Glaser, and D. Sugny, "Time optimal trajectories on $S U(2)$," J. Phys. A: Math. Theor. (submitted).

${ }^{15}$ G. C. Hegerfeldt, "Driving at the quantum speed limit: Optimal control of a two-level system," Phys. Rev. Lett. 111, 260501 (2013).

${ }^{16}$ S. Helgason, Differential Geometry, Lie Groups and Symmetric Spaces (Academic Press, New York, 1978).

${ }^{17}$ R. Huneault, "Time optimal control of closed quantum systems," Master thesis (Department of Mathematics, University of Waterloo, Ontario, Canada, 2009), Available at https://uwspace.uwaterloo.ca/bitstream/handle/10012/4570/Thesis.pdf? sequence $=1$.

${ }^{18}$ V. Jurdjević, Geometric Control Theory, Cambridge Studies in Advanced Mathematics Vol. 52 (Cambridge University Press, 1997).

${ }^{19}$ N. Khaneja, "On some model problems in quantum control," Commun. Inf. Syst. 9(1), 1-40 (2009).

${ }^{20}$ E. Kirillova, T. Hoch, and K. Spindler, "Optimal control of a spin system acting on a single quantum bit," WSEAS Trans. Math. 7, 687 (2008).

${ }^{21}$ M. A. Nielsen and I. L. Chuang, Quantum Computation and Quantum Information (Cambridge University Press, Cambridge, U.K., New York, 2000).

${ }^{22}$ R. Romano, "Geometric analysis of minimum time trajectories for a two level quantum system," Phys. Rev. A 90, 062302 (2014).

${ }^{23}$ K.-H. Spindler, "Optimal control on Lie groups with applications to attitude control," Math. Control, Signals Syst. 11, 197-219 (1998).

${ }^{24}$ Note this terminology is typically used with a slightly different meaning in mathematics, where a separatrix is a curve separating different behaviors of solutions of a differential equation. Here our curves are projections of solutions of differential equations obtained for different values of parameters rather than initial conditions.

${ }^{25}$ Since $\alpha$ is arbitrary in the statement of the proposition, we can modify $\phi$ as we wish without affecting the parameters $\psi$ and $M$ (or $x$ and $y$ ).

${ }^{26}$ The Pontryagin Maximum Principle is described in books on optimal control (see, e.g., Ref. 12). The description we have adopted is the one derived for time optimal control of the evolution of quantum systems in Ref. 17. The relevant result is the 'Complex Matrix Maximum Principle' (Theorem 4.2) in Ref. 17. As compared with the notation in Ref. 17 the Hamiltonian function $H$ in (8) does not contain the constant term $\lambda_{0}$ which we have taken into account by saying that the Hamiltonian function is constant along the optimal trajectory (rather than exactly zero). With this in mind, the form of the Hamiltonian function (8) is a special case of the one obtained for 'Unitary Evolutions' in Theorem 4.4 of Ref. 17, when we take into account Theorem 4.5 ( $M$ there is our $\tilde{M}$ ) and Theorem 4.6 (which allows to remove the Real part sign) of Ref. 17. The final form corresponding to (8) is formula (4.13) in Ref. 17. The description of the maximum principle for systems on Lie groups in a more abstract, coordinate independent, way has been carried out in Refs. 18 and 23 .

${ }^{27}\left[\sigma_{x}, \sigma_{y}\right]=\sigma_{z},\left[\sigma_{y}, \sigma_{z}\right]=\sigma_{x},\left[\sigma_{z}, \sigma_{x}\right]=\sigma_{y}$.

28 The fact that the controls $u_{x}, u_{y}$ are proportional to $b_{x}$ and $b_{y}$ could have also been obtained with the methods of calculus of variations. These were discussed in the context of application to minimum time quantum control in Refs. 9 and 10. In particular, if we assume a constraint of the form $u_{x}^{2}+u_{y}^{2}=\gamma^{2}$, we can obtain $u_{x}=k b_{x}, u_{y}=k b_{y}$ for some $k$, as a result of the Euler-Lagrange equations associated with the variational problem of minimum time with dynamical and static constraints.

${ }^{29}$ If it was equal to zero it would imply $\tilde{M}=0$ which is excluded from the maximum principle.

${ }^{30}$ General conditions to discard singular arcs are discussed in Ref. 3 and the references therein.

${ }^{31}$ This together with the fact that an optimal trajectory does not contain any singular arc, gives an alternative way to prove Proposition 2.1.

${ }^{32}$ In the condition (21) we have used the fact that the time has to be nonnegative (in fact positive if $\psi_{f} \neq 0$ ).

${ }^{33}$ This means that there exists an $\omega$ satisfying (23) with $k=1$ and $m=0$.

${ }^{34}$ Recall that $x_{\psi}:=\frac{\psi-\pi}{\pi}$.

35 The phase on the critical trajectory is an increasing function of $\lambda$ (cf. formula (B8) in Appendix B.) We can simply choose the slope of the line $\psi=\epsilon \lambda$ small enough so that the whole line is below the graph of the function giving the phase.

${ }^{36}$ From the corollary the optimal control cannot be with $\omega \in\left(\omega_{c},+\infty\right)$ since to reach these points the trajectory has to leave the region $Q$ of Figure 5 or being outside of the separatrix, thus loosing optimality. 
${ }^{37}$ This second case can in fact be ruled out by a closer examination of the trajectories but it is quicker to consider the two cases.

${ }^{38}$ Since the correspondence of the Lemma is one to one, if the trajectory crosses the $\epsilon$-curve again it will have to be at a point where another trajectory or itself at a previous time is optimal. Therefore it looses optimality at that point or it was already not optimal.

39 These are defined as $\theta_{1,2,3}$ when writing $x=\cos \left(\theta_{1}\right) \cos \left(\theta_{2}\right), y=\cos \left(\theta_{1}\right) \sin \left(\theta_{2}\right), c=\sin \left(\theta_{1}\right) \cos \left(\theta_{3}\right), d=\sin \left(\theta_{1}\right) \sin \left(\theta_{3}\right)$ (5).

40 Notice that $\left|x_{k, \psi}\right|<1$. By plugging (21) into (22), we obtain $\omega=x_{k, \psi} a$. Using this into the definition $a^{2}:=$ $\gamma^{2}+(1-\omega)^{2}$ we obtain a second order algebraic equation in $a$, whose positive solution is (A8).

${ }^{41}$ It exists because of the existence theorem of optimal control.

${ }^{42}$ To see that $t>\frac{\pi}{2 a}$, one has to adapt the proof of Corollary 4.4 from the critical trajectory to the $\epsilon$-curve. This is easily achieved since we can still apply Lemma 4.2, because the phase of the point on the $\epsilon$-curve is smaller than the phase of the corresponding point on the critical curve. Also, we have chosen $\epsilon$ sufficiently small so that the $\epsilon$-curve is entirely contained in the first quadrant. Optimality is lost at $t=\frac{\pi}{a}$ since the trajectory $P_{\omega}$ touches the boundary of the unit disk.

${ }^{43}$ A rough estimate in Eq. (B17) gives $\pi \leq$ Phase $P_{\omega}(t)$. However since the intersection has to occur in the first quadrant we have $\frac{3 \pi}{2} \leq$ Phase $P_{\omega}(t)$.

${ }^{44}$ We have replaced here in some instances $\omega_{c}, a_{c}$ with their definitions.

${ }^{45}$ It is faster to use the $\omega_{2}$ trajectory or if the time of intersection is the same, this contradicts uniqueness (as well as the fact that the $\omega_{1}$ trajectory is not optimal at $P$ ). 